\title{
The Role of the A-Ring of Bryostatin Analogs in PKC Binding: Synthesis and Initial Biological Evaluation of New A-Ring Modified Bryologs
}

\author{
Paul A. Wender, ${ }^{\star}$ Michael O. Clarke, Joshua C. Horan \\ Department of Chemistry, Stanford University, Stanford, CA 94305-5080
}

Supporting Information

General methods. Air- and moisture-sensitive reactions were carried out in oven-dried glassware sealed with rubber septa under a positive pressure of dry nitrogen, unless otherwise indicated. Similarly, sensitive liquids and solutions were transferred via syringe or stainless steel cannula. Reactions were stirred using Teflon-coated magnetic stir bars. Tetrahydrofuran, diethyl ether, toluene and dicholoromethane were passed through an alumina drying column (Solv-Tek Inc.). Analytical TLC was performed with $0.25 \mathrm{~mm}$ silica gel $60 \mathrm{~F}$ plates with $254 \mathrm{~nm}$ fluorescent indicator from Merck. Plates were visualized by ultraviolet light and treatment with acidic $p$ anisaldehyde stain (unless otherwise indicated) followed by gentle heating. Chromatographic purification of products was accomplished by flash chromatography on silica gel (230-400 mesh; purchased from EM). NMR spectra were measured on a Varian INOVA $600\left({ }^{1} \mathrm{H}\right.$ at $600 \mathrm{MHz},{ }^{13} \mathrm{C}$ at $150 \mathrm{MHz})$, Varian INOVA $500\left({ }^{1} \mathrm{H}\right.$ at $500 \mathrm{MHz},{ }^{13} \mathrm{C}$ at $\left.125 \mathrm{MHz}\right)$, Varian Mercury $400\left({ }^{1} \mathrm{H}\right.$ at $400 \mathrm{MHz},{ }^{13} \mathrm{C}$ at $100 \mathrm{MHz}$ ) or Varian $\mathrm{Gem}-300\left({ }^{1} \mathrm{H}\right.$ at $300 \mathrm{MHz},{ }^{13} \mathrm{C}$ at $\left.75 \mathrm{MHz}\right)$ magnetic resonance spectrometer. Infrared spectra were recorded on a Perkin-Elmer 1600 Series Fourier transform spectrometer (FTIR). Elemental analyses $(\% \mathrm{C}, \% \mathrm{H})$ were performed by Desert Analytics, Tucson, Arizona. Reported atomic percentages are within error limits of $\pm 0.4 \%$. In instances where purity was not determined by elemental analysis, purity is documented via high field ${ }^{1} \mathrm{H}$ NMR spectra showing at most only trace peaks not attributable to the assigned structure. High-resolution mass spectra (HRMS) were recorded at the High Resolution Mass Spectrometry Facility, University of California at Riverside, and at the Mass Spectrometry Facility, University of California at San Francisco.
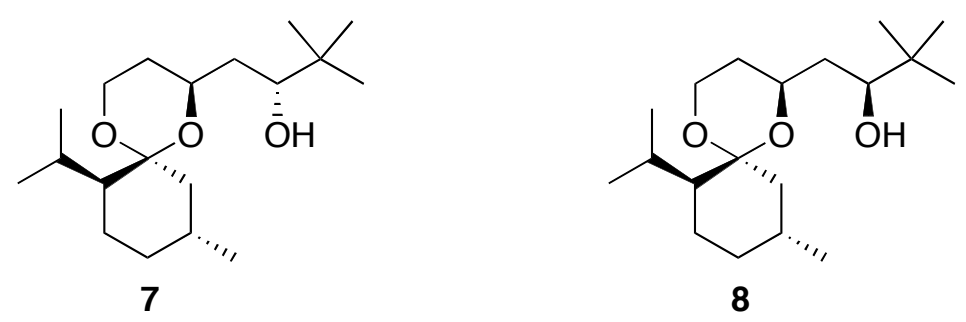

To a stirred solution of aldehyde $6(790 \mathrm{mg}, 3.11 \mathrm{mmol})$ in $15 \mathrm{~mL}$ anhydrous $\mathrm{Et}_{2} \mathrm{O}$ was added $t$-butyllithium $\left(1.7 \mathrm{M}\right.$ in pentane, $2.20 \mathrm{~mL}, 3.73 \mathrm{mmol}$ ) dropwise at $-78^{\circ} \mathrm{C}$. The mixture was stirred for $1 \mathrm{~h}$ between $-78^{\circ} \mathrm{C}$ and $-60^{\circ} \mathrm{C}$ and quenched with saturated aqueous $\mathrm{NH}_{4} \mathrm{Cl}$. The layers were separated and the aqueous phase was extracted with EtOAc (3x). The combined organic extracts were washed with brine, dried over $\mathrm{MgSO}_{4}$, and concentrated in vacuo to afford 
a colorless oil. Purification by flash chromatography $(5 \rightarrow 20 \%$ EtOAc / hexanes) provided 328 $\mathrm{mg}(34 \%)$ of syn-diastereomer 8 and $236 \mathrm{mg}$ (24\%) of anti-diastereomer 7 as colorless oils.

Procedure to recycle 7: To a solution of 7 (142 $\mathrm{mg}, 0.454 \mathrm{mmol})$ in $\mathrm{CH}_{2} \mathrm{Cl}_{2}$ (4 mL) was added powdered $4 \AA$ molecular sieves $(227 \mathrm{mg})$ followed by 4 -methylmorpholine $\mathrm{N}$-oxide $(80$ $\mathrm{mg}, 0.68 \mathrm{mmol}$ ). This suspension was stirred for $5 \mathrm{~min}$ at $\mathrm{rt}$, then the flask was immersed in a $0^{\circ} \mathrm{C}$ bath. Tetrapropylammonium perruthenate $(8.1 \mathrm{mg}, 0.023 \mathrm{mmol})$ was added and the reaction was allowed to stir at $\mathrm{rt}$ for $2 \mathrm{~h}$. The reaction mixture was filtered through a pad of celite, concentrated and then quickly chromatographed on silica (10\% EtOAc / hexanes). The material collected was then dissolved in $\mathrm{MeOH}(8 \mathrm{~mL})$ and $\mathrm{CeCl}_{3} \bullet 7 \mathrm{H}_{2} \mathrm{O}(37 \mathrm{mg}, 0.96 \mathrm{mmol})$ was added. The flask was transferred to a $-78^{\circ} \mathrm{C}$ bath and $\mathrm{NaBH}_{4}(182 \mathrm{mg}, 0.489 \mathrm{mmol})$ was added in one portion. The reaction was allowed to slowly warm to rt over $1 \mathrm{~h}$ and then sat. aq. $\mathrm{NH}_{4} \mathrm{Cl}(6 \mathrm{~mL})$ was added. The mixture was extracted with EtOAc $(3 \times 33 \mathrm{~mL})$ and dried over $\mathrm{MgSO}_{4}$. Flash chromatography on silica gave $111.7 \mathrm{mg}$ of 8 (79\% over 2 steps) and $12.3 \mathrm{mg}$ of 7 (9\% over 2 steps).

Data for 7:

$\mathbf{R}_{f}=0.40$ (15\% EtOAc / hexanes).

IR (thin film): 3507, 2953, 2869, 1456, 1364, 1308, 1267, 1158, 1134, 1102, 1068, $977 \mathrm{~cm}^{-1}$.

${ }^{1} \mathbf{H}$ NMR $\left(300 \mathrm{MHz}, \mathrm{CDCl}_{3}\right): \delta$ 4.01-4.16 (2H, m), $3.81(1 \mathrm{H}, \mathrm{ddd}, J=1.4,5.3,11.5 \mathrm{~Hz}), 3.54$ $(1 \mathrm{H}, \mathrm{dd}, J=1.5,10.2 \mathrm{~Hz}), 2.75(1 \mathrm{H}, \mathrm{ddd}, J=2.0,3.2,13.6 \mathrm{~Hz}), 2.39(1 \mathrm{H}, \mathrm{dsept}, J=1.9,7.1$ $\mathrm{Hz}), 1.14-1.77(11 \mathrm{H}, \mathrm{m}), 0.90(3 \mathrm{H}, \mathrm{d}, J=6.6 \mathrm{~Hz}), 0.89(3 \mathrm{H}, \mathrm{d}, J=6.7 \mathrm{~Hz}), 0.89(9 \mathrm{H}, \mathrm{s}), 0.86$ $(3 \mathrm{H}, \mathrm{d}, J=6.8 \mathrm{~Hz}), 0.70(1 \mathrm{H}, \mathrm{t}, J=12.9 \mathrm{~Hz})$.

${ }^{13}$ C NMR $\left(75 \mathrm{MHz}, \mathrm{CDCl}_{3}\right): \delta 100.6,75.1,64.7,59.1,51.2,38.6,37.4,34.9,34.4,31.9,29.1$, 25.5 (3C), 24.3, 23.8, 22.3, 22.0, 18.7.

HRMS: $\left[\mathrm{M}^{+}\right]$Calculated for $\mathrm{C}_{19} \mathrm{H}_{36} \mathrm{O}_{3}: 312.2664$. Found: 312.2657 .

Elemental Anal.: Calculated for $\mathrm{C}_{19} \mathrm{H}_{36} \mathrm{O}_{3}$ : C, 73.03; H, 11.61. Found: C, 72.57; H, 11.72.

$[\alpha]_{\mathrm{D}}^{28}=+13.7^{\circ}\left(c 1.24, \mathrm{CH}_{2} \mathrm{Cl}_{2}\right)$.

Data for 8:

$\mathbf{R}_{f}=0.73$ (15\% EtOAc / hexanes).

IR (thin film): 3524, 2953, 2870, 1456, 1363, 1309, 1266, 1242, 1188, 1155, 1134, 1092, 973, $953,921,843 \mathrm{~cm}^{-1}$.

${ }^{1}$ H NMR (300 MHz, $\left.\mathrm{CDCl}_{3}\right): \delta$ 4.00-4.18 (2H, m), $3.81(1 \mathrm{H}, \mathrm{ddd}, J=1.1,5.4,11.7 \mathrm{~Hz}), 3.58$ $(1 \mathrm{H}, \mathrm{s}, \mathrm{OH}), 3.44(1 \mathrm{H}, \mathrm{d}, J=9.3 \mathrm{~Hz}), 2.75(1 \mathrm{H}, \mathrm{ddd}, J=1.7,2.8,13.6 \mathrm{~Hz}), 2.41(1 \mathrm{H}, b r$ sept, $J=$ $6.3 \mathrm{~Hz}), 1.17-1.81(10 \mathrm{H}, \mathrm{m}), 0.92(3 \mathrm{H}, \mathrm{d}, J=6.7 \mathrm{~Hz}), 0.90(6 \mathrm{H}, \mathrm{d}, J=6.8 \mathrm{~Hz}), 0.89(9 \mathrm{H}, \mathrm{s}), 0.75$ $(1 \mathrm{H}, \mathrm{t}, J=13.2 \mathrm{~Hz})$. 
${ }^{13} \mathrm{C}$ NMR $\left(75 \mathrm{MHz}, \mathrm{CDCl}_{3}\right): \delta 101.1,80.1,70.5,58.8,51.0,37.8,37.3,34.6,34.5,31.9,28.9$, $25.6(3 \mathrm{C}), 24.2,23.7,22.3,22.2,18.9$.

HRMS: [ $\left.\mathrm{M}^{+}\right]$Calculated for $\mathrm{C}_{19} \mathrm{H}_{36} \mathrm{O}_{3}: 312.2664$. Found: 312.2659 .

Elemental Anal.: Calculated for $\mathrm{C}_{19} \mathrm{H}_{36} \mathrm{O}_{3}: \mathrm{C}, 73.03 ; \mathrm{H}, 11.61$. Found: $\mathrm{C}, 73.15 ; \mathrm{H}, 11.41$.

$[\alpha]_{\mathrm{D}}^{28}=-1.6^{\circ}\left(c 0.69, \mathrm{CH}_{2} \mathrm{Cl}_{2}\right)$.

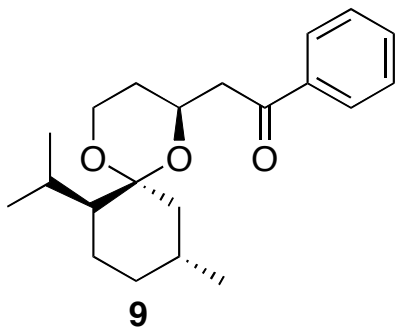

To a solution of aldehyde $6(77.2 \mathrm{mg}, 0.303 \mathrm{mmol})$ in $\mathrm{CH}_{2} \mathrm{Cl}_{2}(0.75 \mathrm{~mL})$ under $\mathrm{N}_{2}$, at $-78^{\circ} \mathrm{C}$ (external temp.) was added $330 \mu \mathrm{L}$ of $\mathrm{PhMgBr}(1 \mathrm{M}$ solution in THF) via syringe. The clear solution was stirred and allowed to warm to $0^{\circ} \mathrm{C}$ over $3 \mathrm{hr}$. The reaction was maintained at $0^{\circ} \mathrm{C}$ for $2 \mathrm{hr}$ then quenched by sequential addition of $1 \mathrm{~mL}$ sat. $\mathrm{NH}_{4} \mathrm{Cl}(\mathrm{aq}), 1 \mathrm{~mL}$ brine and $1 \mathrm{~mL}$ $\mathrm{H}_{2} \mathrm{O}$. This mixture was extracted with EtOAc $(4 \times 2 \mathrm{~mL})$. The combined organic layers were dried with anhydrous $\mathrm{MgSO}_{4}$, filtered and concentrated. The residue was filtered through a 2" pad of silica using 10\% EtOAc/pentane to remove baseline material. The solvent was removed in vacuo to give a crude mixture of diastereomeric alcohols as a pale yellow oil.

To a solution of the crude mixture in $\mathrm{CH}_{2} \mathrm{Cl}_{2}(8 \mathrm{~mL})$, under $\mathrm{N}_{2}$, at $0^{\circ} \mathrm{C}$ (external temp.) was added Dess-Martin Periodinane (206 $\mathrm{mg}, 0.486 \mathrm{mmol}$ ). The flask was allowed to warm to $\mathrm{rt}$ and stir for $1 \mathrm{hr}$. Saturated $\mathrm{NaHCO}_{3}(\mathrm{aq})(2 \mathrm{~mL})$ and sat. $\mathrm{Na}_{2} \mathrm{~S}_{2} \mathrm{O}_{3}(\mathrm{aq})(5 \mathrm{~mL})$ were added and stirring was continued for $1 \mathrm{hr}$. The biphasic mixture was separated and the aqueous layer was washed with EtOAc $(3 \times 5 \mathrm{~mL})$. The combined organic layers were dried with anhydrous $\mathrm{MgSO}_{4}$, filtered and concentrated. Flash chromatography of the resulting oil on silica gel (5\% EtOAc/pentane) gave $89.6 \mathrm{mg}$ of ketone $\mathbf{9}$ as a clear, colorless oil ( $84 \%$ over 2 steps).

Data for 9:

$\mathbf{R}_{f}=0.66$ (10\% EtOAc, $90 \%$ hexanes).

IR (thin film): 3061, 2952, 2869, 1688, 1598, 1449, 1373, 1307, 1265, 1157, 1131, 1100, 975, $801,754,690 \mathrm{~cm}^{-1}$.

${ }^{1} \mathbf{H}$ NMR $\left(500 \mathrm{MHz}, \mathrm{CDCl}_{3}\right): \delta$ 7.46-7.95 $(5 \mathrm{H}, \mathrm{m}), 4.46(1 \mathrm{H}, \mathrm{m}), 4.15(1 \mathrm{H}, \mathrm{dt}, J=3.0,12.0 \mathrm{~Hz})$, $3.83(1 \mathrm{H}, \mathrm{ddd}, J=1.6,5.2,11.6 \mathrm{~Hz}), 3.29(1 \mathrm{H}, \mathrm{dd}, J=7.2,15.7 \mathrm{~Hz}), 2.84(1 \mathrm{H}, \mathrm{dd}, J=5.3,15.7$ $\mathrm{Hz}), 2.72(1 \mathrm{H}, \mathrm{ddd}, J=2.0,3.5,12.6 \mathrm{~Hz}), 2.39(1 \mathrm{H}, \mathrm{dsept}, J=2.0,7.0 \mathrm{~Hz}), 1.68(1 \mathrm{H}, \mathrm{ddt}, J=$ $5.3,11.3,12.5 \mathrm{~Hz}), 1.60(2 \mathrm{H}, \mathrm{m}), 1.34(2 \mathrm{H}, \mathrm{m}), 1.18(2 \mathrm{H}, \mathrm{m}), 0.89(3 \mathrm{H}, \mathrm{d}, J=7.0 \mathrm{~Hz}), 0.87(3 \mathrm{H}$, $\mathrm{d}, J=7.0 \mathrm{~Hz}), 0.86(3 \mathrm{H}, \mathrm{d}, J=6.7 \mathrm{~Hz}), 0.79(1 \mathrm{H}, \mathrm{m}), 0.66(1 \mathrm{H}, \mathrm{dd}, J=12.6,13.5 \mathrm{~Hz})$. 
${ }^{13}$ C NMR (125 MHz, $\left.\mathrm{CDCl}_{3}\right): \delta 198.9,137.5,133.0,128.4(\mathrm{x} 2), 128.2(\mathrm{x} 2), 100.8,65.5,58.9$, $51.1,45.4,37.3,34.8,31.7,28.5,24.3,23.8,22.3,21.6,18.9$.

HRMS: $\left[\mathrm{M}^{+}\right]$Calculated for $\mathrm{C}_{21} \mathrm{H}_{30} \mathrm{O}_{3}: 330.2195$. Found: 330.2187 .

$[\alpha]_{D}^{25}=-9.4^{\circ}\left(c 0.16, \mathrm{CDCl}_{3}\right)$.

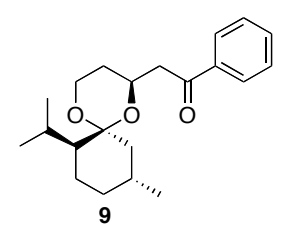

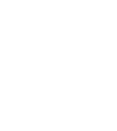
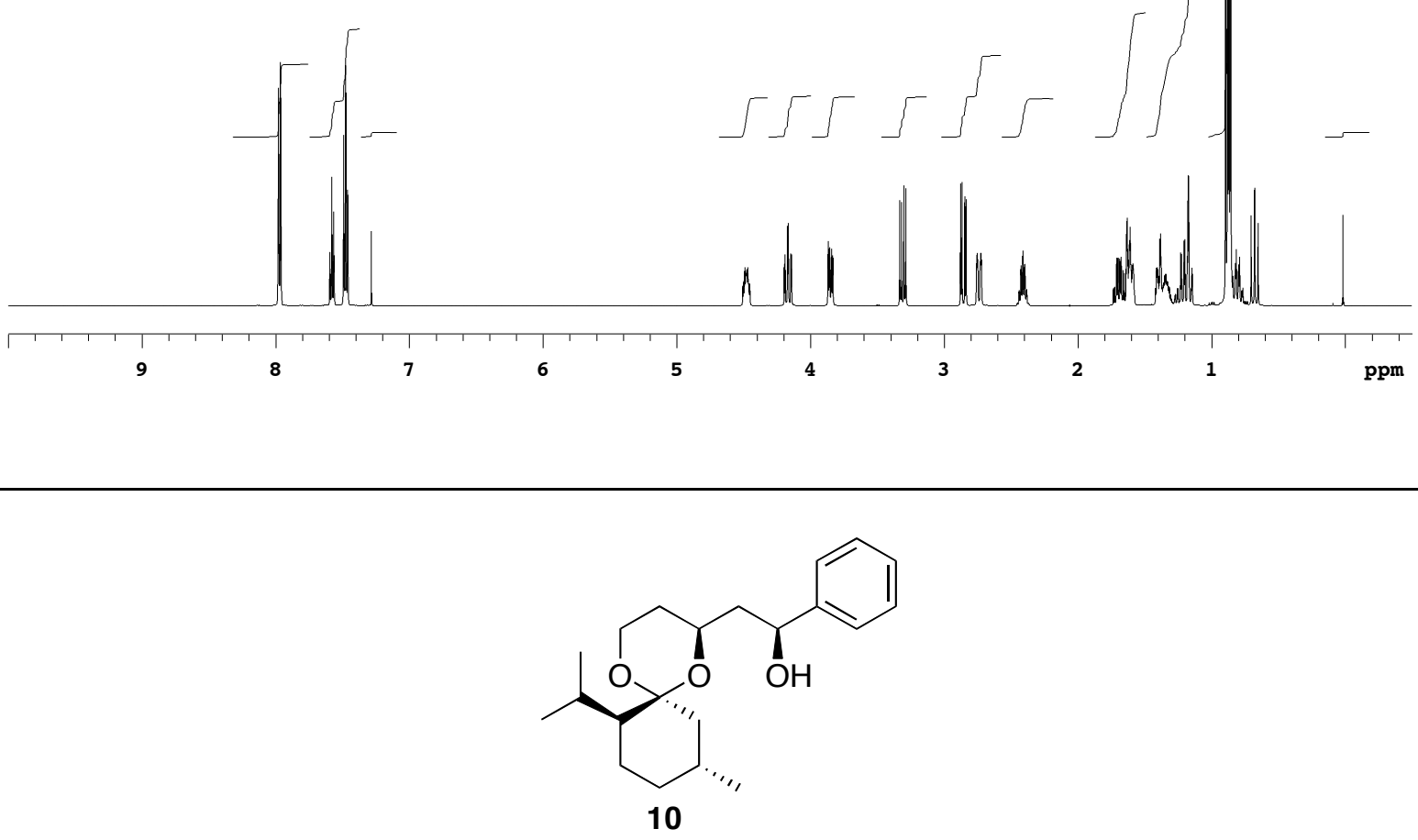

To a solution of ketone $9(89.6 \mathrm{mg}, 0.271 \mathrm{mmol})$ in $\mathrm{MeOH}(5 \mathrm{~mL})$, under $\mathrm{N}_{2}$, at $-78^{\circ} \mathrm{C}$ (external temp.) was added $\mathrm{CeCl}_{3} \bullet 7 \mathrm{H}_{2} \mathrm{O}(111 \mathrm{mg}, 0.298 \mathrm{mmol})$ followed by $\mathrm{NaBH}_{4}(22 \mathrm{mg}$, $0.583 \mathrm{mmol})$. The reaction was allowed to stir at $-78^{\circ} \mathrm{C}$ for $1 \mathrm{hr}$; the cold bath was removed and sat. aq. $\mathrm{NaHCO}_{3}(5 \mathrm{~mL})$ and $\mathrm{H}_{2} \mathrm{O}(5 \mathrm{~mL})$ were added. The reaction mixture was extracted with EtOAc $(3 \times 15 \mathrm{~mL})$ and the combined organic layers were dried with anhydrous $\mathrm{MgSO}_{4}$, filtered and concentrated. Flash chromatography of the resulting oil on silica (10\% EtOAc/pentane) gave $70.6 \mathrm{mg}$ of $\mathbf{1 0}$ as a pale yellow oil (78\%). 
Data for 10:

$\mathbf{R}_{f}=0.54$ (15\% EtOAc, $85 \%$ pentane).

IR (thin film): 3494, 3063, 3030, 2951, 2869, 1947, 1879, 1811, 1604, 1495, 1454, 1377, 1307, $1268,1240,1218,1201,1155,1136,1101,1066,1029,1003 \mathrm{~cm}^{-1}$;

${ }^{1} \mathbf{H}$ NMR $\left(500 \mathrm{MHz}, \mathrm{CDCl}_{3}\right): \delta$ 7.23-7.41 $(5 \mathrm{H}, \mathrm{m}), 4.94(1 \mathrm{H}, \mathrm{dd}, J=2.3,9.8 \mathrm{~Hz}), 4.22(1 \mathrm{H}, \mathrm{m})$, $4.15(1 \mathrm{H}$, ddd, $J=2.6,11.6,12.7 \mathrm{~Hz}), 4.04(1 \mathrm{H}, \mathrm{s}), 3.83(1 \mathrm{H}, \mathrm{ddd}, J=1.2,5.3,11.6 \mathrm{~Hz}), 2.79$ $(1 \mathrm{H}, \mathrm{ddd}, J=2.4,3.0,13.7 \mathrm{~Hz}), 2.46(1 \mathrm{H}, \mathrm{dsept}, J=1.9,6.9 \mathrm{~Hz}), 1.92(1 \mathrm{H}, \mathrm{dt}, J=14.5,9.9 \mathrm{~Hz})$, $1.79(2 \mathrm{H}, \mathrm{m}), 1.70(1 \mathrm{H}, \mathrm{ddt}, J=5.4,11.5,12.9 \mathrm{~Hz}), 1.61(2 \mathrm{H}, \mathrm{m}), 1.41(1 \mathrm{H}, \mathrm{m}), 1.35(1 \mathrm{H}, \mathrm{dq}, J$ $=3.5,13.1 \mathrm{~Hz}), 1.27(1 \mathrm{H}, \mathrm{m}), 0.97(3 \mathrm{H}, \mathrm{d}, J=7.0 \mathrm{~Hz}), 0.95(3 \mathrm{H}, \mathrm{d}, J=6.6 \mathrm{~Hz}), 0.93(3 \mathrm{H}, \mathrm{d}, J=$ $7.0 \mathrm{~Hz}), 0.91(1 \mathrm{H}, \mathrm{m}), 0.79(1 \mathrm{H}, \mathrm{t}, J=13.2 \mathrm{~Hz})$.

${ }^{13}$ C NMR (75 MHz, $\left.\mathrm{CDCl}_{3}\right):$ 144.1, 128.3 (x2), 127.3, 125.7 (x2), 101.1, 74.4, 69.8, 58.9, 51.0, $45.7,37.3,34.6,31.6,29.1,24.3,23.8,22.3,22.2$, 18.9.

HRMS: $\left[\mathrm{M}^{+}\right]$Calculated for $\mathrm{C}_{21} \mathrm{H}_{32} \mathrm{O}_{3}$ : 332.2351. Found: 332.2359.

Elemental Anal.: Calculated for $\mathrm{C}_{21} \mathrm{H}_{32} \mathrm{O}_{3}$ : C 75.86; H 9.70. Found C 75.60; $\mathrm{H} 10.00$.

$[\alpha]_{D}^{25}=-29.6^{\circ}\left(c 3.25, \mathrm{CH}_{2} \mathrm{Cl}_{2}\right)$.

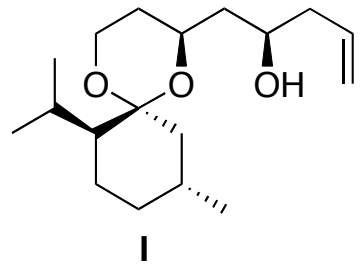

A stock solution of (+)-Ipc $\mathrm{I}_{2} \mathrm{~B}$ (allyl) was prepared by dissolving (+)-Ipc $\mathrm{IOMe}_{2}(1.99 \mathrm{~g}$, $6.3 \mathrm{mmol})$ in $\mathrm{Et}_{2} \mathrm{O}(18 \mathrm{~mL})$ in $50 \mathrm{~mL}$ round bottom flask. The flask was immersed in a dry ice/acetone bath and allylmagnesium bromide (1 M in THF, $5.2 \mathrm{~mL}, 5.2 \mathrm{mmol}$ ) was added dropwise. The dry ice/acetone bath was then removed and the solution was allowed to warm to rt. After stirring for $1 \mathrm{~h}$ at $\mathrm{rt}$ stirring was stopped. In a separate $50 \mathrm{~mL}$ round bottom flask 6 (242 $\mathrm{mg}, 0.951 \mathrm{mmol})$ was dissolved in $\mathrm{Et}_{2} \mathrm{O}(30 \mathrm{~mL})$. This flask was cooled in a dry ice/acetone bath and the solution of $(+)-\mathrm{Ipc}_{2} \mathrm{~B}$ (allyl) $(6.61 \mathrm{~mL}, 1.48 \mathrm{mmol})$ was added drop-wise. Upon complete addition, the reaction was allowed to stir for $3 \mathrm{~h}$ immersed in the dry ice/acetone bath. $3 \mathrm{M}$ $\mathrm{NaOH}_{\text {(aq.) }}(2.3 \mathrm{~mL})$ and $30 \%(\mathrm{w} / \mathrm{w}) \mathrm{H}_{2} \mathrm{O}_{2 \text { (aq.) }}(0.96 \mathrm{~mL})$ were then added to the reaction and then the cold bath was removed. The reaction was stirred for another $3.5 \mathrm{~h}$ and excess $\mathrm{H}_{2} \mathrm{O}_{2}$ was quenched by the addition of a saturated solution of $\mathrm{Na}_{2} \mathrm{~S}_{2} \mathrm{O}_{3 \text { (aq.) }}(5 \mathrm{~mL})$. The organic phase was diluted with $\mathrm{Et}_{2} \mathrm{O}(40 \mathrm{~mL})$ and the organic phase was then extracted with brine $(2 \times 40 \mathrm{~mL})$. The organic phase was dried over $\mathrm{MgSO}_{4}$. After removal of the $\mathrm{MgSO}_{4}$ by vacuum filtration, the filtrate was concentrated in vacuo and the homoallylic alcohol $\mathbf{I}$ was isolated from the residue by flash chromatography (silica gel, 3:17 EtOAc/pentane) as a clear oil (265 mg, 94\%).

Data for $\mathbf{I}$ : 
$\mathbf{R}_{f}=0.39(15 \%$ EtOAc / pentane $)$.

${ }^{1}$ H NMR $\left(500 \mathrm{MHz}, \mathrm{CDCl}_{3}\right): \delta 5.83(1 \mathrm{H}, \mathrm{m}), 5.08(2 \mathrm{H}, \mathrm{m}), 4.11(1 \mathrm{H}, \mathrm{td}, J=3,13 \mathrm{~Hz}), 4.08$ $(1 \mathrm{H}, \mathrm{m}), 3.89(1 \mathrm{H}, \mathrm{m}), 3.80(1 \mathrm{H}, \mathrm{ddd}, J=1,6.5,12 \mathrm{~Hz}), 3.72(1 \mathrm{H}, \mathrm{s}), 2.74(1 \mathrm{H}, \mathrm{ddd}, J=2,3$, $13.5 \mathrm{~Hz}), 2.40(1 \mathrm{H}$, sept, $J=7 \mathrm{~Hz}), 2.28(1 \mathrm{H}$, pent, $J=7 \mathrm{~Hz}), 2.16(1 \mathrm{H}$, pent, $J=7 \mathrm{~Hz}), 1.74$ $(1 \mathrm{H}, \mathrm{brd}, J=13 \mathrm{~Hz}), 1.67(1 \mathrm{H}, \mathrm{qd}, J=5.5,13 \mathrm{~Hz}), 1.52-1.63(3 \mathrm{H}, \mathrm{m}), 1.48(1 \mathrm{H}, \mathrm{m}), 1.39(1 \mathrm{H}$, ddd, $J=2.5,4,13 \mathrm{~Hz}), 1.24(2 \mathrm{H}, \mathrm{m}), 0.85-0.93(10 \mathrm{H}, \mathrm{m}), 0.74(1 \mathrm{H}, \mathrm{t}, J=13.5 \mathrm{~Hz})$.

${ }^{13}$ C NMR $\left(125 \mathrm{MHz}, \mathrm{CDCl}_{3}\right): \delta 134.9,117.2,101.0,71.7,70.0,58.9,50.9,42.3,41.9,37.2$, 34.6, 31.7, 29.0, 24.2, 23.7, 22.3, 22.1, 18.9 .

IR (thin film): 3524, 2952, 2870, 1641, 1456, 1430, 1375, 1309, 1268, 1156, 1141, 1121, 974 , $913,849 \mathrm{~cm}^{-1}$.

HRMS: [ $\left.\mathrm{M}^{+}\right]$Calculated for $\mathrm{C}_{18} \mathrm{H}_{32} \mathrm{O}_{3}: 296.2351$; found: 296.2359 .

Elemental Anal.: Calculated for $\mathrm{C}_{18} \mathrm{H}_{32} \mathrm{O}_{3}$ : C, $72.93 ; \mathrm{H}, 10.88$; found: $\mathrm{C}, 72.61 ; \mathrm{H}, 11.11$. $[\alpha]_{\mathrm{D}}^{25}=+5.4^{\circ}\left(c 1.57, \mathrm{CH}_{2} \mathrm{Cl}_{2}\right)$.

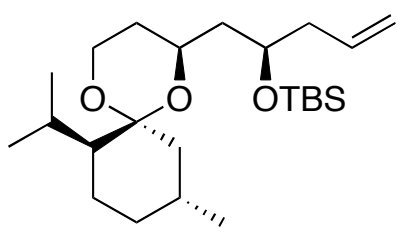

11

In a $25 \mathrm{~mL}$ round bottom flask $\mathbf{I}(229 \mathrm{mg}, 0.77 \mathrm{mmol})$ was dissolved in DMF $(6.5 \mathrm{~mL})$. To this solution was added TBSCl (232 g, $1.54 \mathrm{mmol})$ and imidazole $(131 \mathrm{mg}, 1.93 \mathrm{mmol})$. The reaction was allowed to stir for $3.5 \mathrm{~h}$. The reaction was diluted with pentane $(100 \mathrm{~mL})$ and then the organic phase was extracted with saturated $\mathrm{NH}_{4} \mathrm{Cl}_{\text {(aq.) }}(2 \times 100 \mathrm{~mL})$, saturated $\mathrm{NaHCO}_{3 \text { (aq.) }}(1$ x $100 \mathrm{~mL})$, and brine $(1 \times 100 \mathrm{~mL})$. The organic phase was then dried over $\mathrm{MgSO}_{4}$, which was subsequently removed by vacuum filtration. The filtrate was concentrated in vacuo and $\mathbf{1 1}$ was isolated from the residue by flash chromatography (silica gel, 1/19 $\mathrm{Et}_{2} \mathrm{O}$ :pentane) as a clear oil (287 mg, 91\%).

Data for 11:

$\mathbf{R}_{f}=0.53\left(5 \% \mathrm{Et}_{2} \mathrm{O} /\right.$ pentane $)$.

${ }^{1}$ H NMR $\left(500 \mathrm{MHz}, \mathrm{CDCl}_{3}\right): \delta 5.85(1 \mathrm{H}, \mathrm{m}), 5.03(2 \mathrm{H}, \mathrm{m}), 4.08(1 \mathrm{H}, \mathrm{td}, J=2.5,13 \mathrm{~Hz}), 3.90$ $(2 \mathrm{H}, \mathrm{m}), 3.80(1 \mathrm{H}, \mathrm{ddd}, J=1.5,5.5,11.5 \mathrm{~Hz}), 2.70(1 \mathrm{H}, \mathrm{ddd}, J=2,3,13.5 \mathrm{~Hz}), 2.41\left(1 \mathrm{H}_{\mathrm{F}} \mathrm{dsept}\right.$, $J=7,1.5 \mathrm{~Hz}), 2.31(1 \mathrm{H}, \mathrm{m}), 2.19(1 \mathrm{H}, \mathrm{m}), 1.64-1.75(2 \mathrm{H}, \mathrm{m}), 1.56(1 \mathrm{H}, \mathrm{qd}, J=5.5,11.5 \mathrm{~Hz})$, $1.47-1.54(3 \mathrm{H}, \mathrm{m}), 1.43(1 \mathrm{H}, \mathrm{td}, J=3.5,13 \mathrm{~Hz}), 1.36(1 \mathrm{H}, \mathrm{brd}, J=13 \mathrm{~Hz}), 1.18(1 \mathrm{H}, \mathrm{dq}, J=2$, $12.5 \mathrm{~Hz}), 0.86-0.92(19 \mathrm{H}, \mathrm{m}), 0.69(1 \mathrm{H}, \mathrm{t}, J=13 \mathrm{~Hz}), 0.04(6 \mathrm{H}, \mathrm{d}, J=5.5 \mathrm{~Hz})$. 
${ }^{13}$ C NMR $\left(125 \mathrm{MHz}, \mathrm{CDCl}_{3}\right): \delta 135.6,116.6,100.5,68.5,64.64,59.2,51.3,43.9,41.4,37.4$, $35.0,32.0,29.2,25.8$ (3C), 24.3, 23.8, 22.2, 21.9, 18.9, 18.0, -4.5, -4.6.

IR (thin film) : 2952, 2868, 1641, 1472, 1459, 1374, 1361, 1308, 1256, 1219, 1143, 1112, 1073, $1052,1004,978,913,836,775 \mathrm{~cm}^{-1}$.

HRMS: [ $\left.\mathrm{M}^{+}\right]$Calculated for $\mathrm{C}_{24} \mathrm{H}_{46} \mathrm{O}_{3} \mathrm{Si}$ : 410.3216; found: 410.3225 .

Elemental Anal.: Calculated for $\mathrm{C}_{24} \mathrm{H}_{46} \mathrm{O}_{3} \mathrm{Si}$ : C, $70.19 ; \mathrm{H}, 11.29$; found: $\mathrm{C}, 70.21 ; \mathrm{H}, 11.48$.

$[\alpha]_{\mathrm{D}}^{25}=-6.4^{\circ}\left(c 1.80, \mathrm{CH}_{2} \mathrm{Cl}_{2}\right)$.

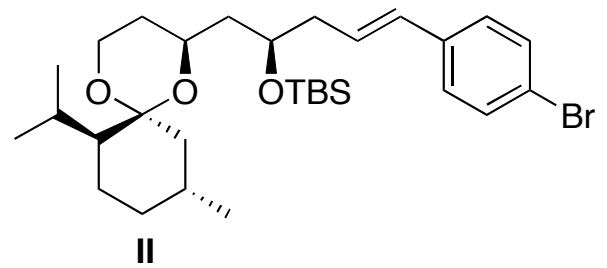

In a $25 \mathrm{~mL}$ round bottom flask $11(298 \mathrm{mg}, 0.726 \mathrm{mmol})$ was dissolved in 1, 2dichloroethane $(3.3 \mathrm{~mL})$. $p$-bromostyrene $(209 \mu \mathrm{L}, 1.60 \mathrm{mmol})$ was then added in one portion followed by Grubb's $2^{\text {nd }}$ Generation Catalyst ( $38 \mathrm{mg}, 0.044 \mathrm{mmol}$ ). The reaction vessel was then placed in a preheated $40^{\circ} \mathrm{C}$ oil bath and stirred for $1 \mathrm{~h}$. After $1 \mathrm{~h}$ more Grubb's $2^{\text {nd }}$ Generation Catalyst $(20 \mathrm{mg}, 0.024 \mathrm{mmol})$ and more $p$-bromostyrene $(200 \mu \mathrm{L}, 1.53 \mathrm{mmol})$ was added and the reaction was stirred for another $1 \mathrm{~h}$ at $40^{\circ} \mathrm{C}$. More Grubb's $2^{\text {nd }}$ Generation Catalyst $(20 \mathrm{mg}, 0.024$ $\mathrm{mmol})$ and more $p$-bromostyrene $(200 \mu \mathrm{L}, 1.53 \mathrm{mmol})$ were added and the reaction was stirred for $1 \mathrm{~h}$ more at $40^{\circ} \mathrm{C}$. The reaction was then cooled to rt and diluted with $\mathrm{Et}_{2} \mathrm{O}(50 \mathrm{~mL})$. The organic phase was extracted with saturated $\mathrm{NH}_{4} \mathrm{Cl}_{\text {(aq.) }}(1 \times 50 \mathrm{~mL})$ and brine $(1 \times 50 \mathrm{~mL})$. The organic phase was then dried over $\mathrm{MgSO}_{4}$, which was then removed by vacuum filtration. The filtrate was then concentrated in vacuo and II was isolated from the residue by flash chromatography (silica gel 1/19 $\mathrm{Et}_{2} \mathrm{O}$ :pentane) as a clear oil (241 mg, 59\%).

Data for II:

$\mathbf{R}_{f}=0.27\left(5 \% \mathrm{Et}_{2} \mathrm{O} /\right.$ pentane $)$.

${ }^{1} \mathbf{H}$ NMR $\left(500 \mathrm{MHz}, \mathrm{CDCl}_{3}\right): \delta 7.42(2 \mathrm{H}, \mathrm{d}, J=8.5 \mathrm{~Hz}), 7.20(2 \mathrm{H}, \mathrm{d}, J=8.5 \mathrm{~Hz}), 6.28(2 \mathrm{H}, \mathrm{m})$, $4.09(1 \mathrm{H}, \mathrm{td}, J=2.5,12.5 \mathrm{~Hz}), 4.97(2 \mathrm{H}, \mathrm{m}), 3.82(1 \mathrm{H}, \mathrm{ddd}, J=1.5,5,11.5 \mathrm{~Hz}), 2.73(1 \mathrm{H}, \mathrm{brd}, J$ $=13.5 \mathrm{~Hz}), 2.44(1 \mathrm{H}$, dsept, $J=7,1.5 \mathrm{~Hz}), 2.45(1 \mathrm{H}$, pent, $J=7 \mathrm{~Hz}), 2.33(1 \mathrm{H}$, pent, $J=7 \mathrm{~Hz})$, $1.69-1.77(2 \mathrm{H}, \mathrm{m}), 1.58(1 \mathrm{H}, \mathrm{qd}, J=5.5,13 \mathrm{~Hz}), 1.48-1.56(3 \mathrm{H}, \mathrm{m}), 1.43(1 \mathrm{H}, \mathrm{qd}, J=3.5,13$ $\mathrm{Hz}), 1.37(1 \mathrm{H}, \mathrm{dd}, J=1.5,13 \mathrm{~Hz}), 1.20(1 \mathrm{H}, \mathrm{dq}, J=2,13 \mathrm{~Hz}), 0.87-0.95(19 \mathrm{H}, \mathrm{m}), 0.71(1 \mathrm{H}, \mathrm{t}, J$ $=13 \mathrm{~Hz}), 0.03(6 \mathrm{H}, \mathrm{d}, J=4.5 \mathrm{~Hz})$.

${ }^{13} \mathrm{C}$ NMR (125 MHz, $\left.\mathrm{CDCl}_{3}\right): \delta 136.6,131.6$ (2 C), 130.7, 128.5, 127.5 (2C), 120.6, 100.5, 68.7, 64.6, 59.2, 51.3, 44.0, 40.5, 37.4, 35.0, 32.0, 29.2, 25.8 (3C), 24.3, 23.8, 22.3, 21.9, 19.0, 18.0, 4.5, -4.6 . 
IR (thin film): 2952, 2867, 1488, 1461, 1361, 1307, 1256, 1218, 1157, 1110, 1073, 1008, 972, $837,775,735,668 \mathrm{~cm}^{-1}$.

HRMS: [ $\left.\mathrm{M}^{+}\right]$Calculated for $\mathrm{C}_{30} \mathrm{H}_{49} \mathrm{BrO}_{3} \mathrm{Si}$ : 564.2634; found: 564.2636.

Elemental Anal.: Calculated for $\mathrm{C}_{30} \mathrm{H}_{49} \mathrm{BrO}_{3} \mathrm{Si}$ : C, 63.69; H, 8.73; found: $\mathrm{C}, 63.81 ; \mathrm{H}, 8.90$.

$[\alpha]_{\mathrm{D}}^{25}=+10.6^{\circ}\left(c 1.16, \mathrm{CH}_{2} \mathrm{Cl}_{2}\right)$.

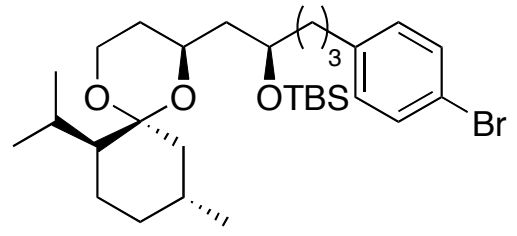

12

In a $200 \mathrm{~mL}$ round bottom flask II $(255 \mathrm{mg}, 0.421 \mathrm{mmol})$ was dissolved in EtOH (98 $\mathrm{mL}$ ). To this solution was added $5 \% \mathrm{Rh} / \mathrm{Al}_{2} \mathrm{O}_{3}$ and the reaction flask was then purged under vacuum, inducing bubbling of the reaction solution, for $1 \mathrm{~min}$. The reaction flask was then repressurized with $\mathrm{H}_{2}$ gas and the reaction was allowed to stir under the hydrogen atmosphere for 1 $\min$. The evacuation/re-pressurization procedure was repeated 2 more times and then the reaction was allowed to stir for $5 \mathrm{~h}$. The $\mathrm{H}_{2}$ atmosphere was exchanged for $\mathrm{N}_{2}$ and then the reaction was filtered through a plug of celite, eluting with $\mathrm{Et}_{2} \mathrm{O}$. The filtrate was concentrated in vacuo and $\mathbf{1 2}$ was isolated from the residue by flash chromatography (silica gel 1/19 $\mathrm{Et}_{2} \mathrm{O}$ :pentane) as a clear oil (188 mg, 73\%).

Data for 12:

$\mathbf{R}_{f}=0.35\left(5 \% \mathrm{Et}_{2} \mathrm{O} /\right.$ pentane $)$.

${ }^{1} \mathbf{H}$ NMR $\left(500 \mathrm{MHz}, \mathrm{CDCl}_{3}\right): \delta 7.39(2 \mathrm{H}, \mathrm{d}, J=8.5 \mathrm{~Hz}), 7.04(2 \mathrm{H}, \mathrm{d}, J=8 \mathrm{~Hz}), 4.06(1 \mathrm{H}, \mathrm{td}, J=$ $2.5,12.5 \mathrm{~Hz}), 3.85(2 \mathrm{H}, \mathrm{m}), 3.79(1 \mathrm{H}, \mathrm{ddd}, J=1.5,5.5,11.5 \mathrm{~Hz}), 2.66(1 \mathrm{H}, \operatorname{brd}, J=13 \mathrm{~Hz}), 2.55$ $(2 \mathrm{H}, \mathrm{m}), 2.39(1 \mathrm{H}, \mathrm{dsept}, J=7,1.5 \mathrm{~Hz}), 1.63-1.74(2 \mathrm{H}, \mathrm{m}), 1.31-1.63(10 \mathrm{H}, \mathrm{m}), 1.17(1 \mathrm{H}, \mathrm{dq}, J$ $=2,12.5 \mathrm{~Hz}), 0.85-0.91(19 \mathrm{H}, \mathrm{m}), 0.67(1 \mathrm{H}, \mathrm{t}, J=13 \mathrm{~Hz}), 0.02(6 \mathrm{H}, \mathrm{s})$.

${ }^{13} \mathrm{C}$ NMR $\left(125 \mathrm{MHz}, \mathrm{CDCl}_{3}\right): \delta 141.5,131.3$ (2C), 130.1 (2C), 119.3, 100.4, 68.5, 64.6, 59.2, $51.2,44.0,37.4,36.2,35.5,34.9,32.0,29.1,26.9,25.8$ (3C), 24.3, 23.7, 22.2, 21.9, 18.9, 18.0, $4.48,-4.53$.

IR (thin film): 2951, 2866, 1488, 1456, 1375, 1307, 1256, 1143, 1113, 1073, 1012, 977, 835, $774 \mathrm{~cm}^{-1}$.

HRMS: [ $\left.\mathrm{M}^{+}\right]$Calculated for $\mathrm{C}_{30} \mathrm{H}_{51} \mathrm{BrO}_{3} \mathrm{Si}$ : 566.2791; found: 566.2800 .

Elemental Anal.: Calculated for $\mathrm{C}_{30} \mathrm{H}_{51} \mathrm{BrO}_{3} \mathrm{Si}$ : C, 63.47; H, 9.05; found: C, 63.42; H, 8.92.

$[\alpha]_{\mathrm{D}}^{25}=+6.6^{\circ}\left(c 1.51, \mathrm{CH}_{2} \mathrm{Cl}_{2}\right)$. 


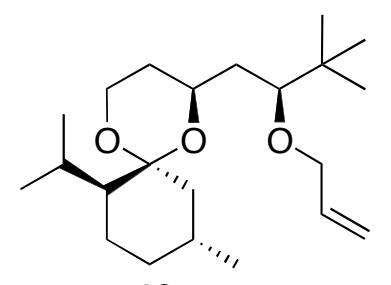

13

To a stirred solution of alcohol $8(320 \mathrm{mg}, 1.02 \mathrm{mmol})$ in $3 \mathrm{~mL}$ anhydrous THF was added potassium $t$-butoxide $(172 \mathrm{mg}, 1.538 \mathrm{mmol})$ in a single portion at $\mathrm{rt}$. The mixture was stirred for $30 \mathrm{~min}$ at $\mathrm{rt}$, treated with allyl bromide $(131 \mu \mathrm{L}, 183 \mathrm{mg}, 1.538 \mathrm{mmol})$ and mixed for an additional $19 \mathrm{~h}$. The reaction was diluted with $\mathrm{Et}_{2} \mathrm{O}(70 \mathrm{~mL})$ and washed with saturated aqueous $\mathrm{NH}_{4} \mathrm{Cl}$ and brine. The organic layer was dried over $\mathrm{MgSO}_{4}$, filtered and to give a colorless oil. Flash chromatography $(3 \rightarrow 10 \%$ EtOAc / hexanes) affords allyl ether 13 (184 mg, $51 \%)$ as well as recovered $\mathbf{8}(132 \mathrm{mg}, 41 \%)$.

Data for 13:

$\mathbf{R}_{f}=0.83$ (15\% EtOAc / hexanes).

IR (thin film): 2953, 2869, 1456, 1375, 1362, 1307, 1267, 1158, 1139, 1109, 1093, 1054, 975, $957,918 \mathrm{~cm}^{-1}$.

${ }^{1} \mathbf{H}$ NMR $\left(300 \mathrm{MHz}, \mathrm{CDCl}_{3}\right): \delta 5.91(1 \mathrm{H}, \mathrm{dddd}, J=5.3,5.3,10.5,17.2 \mathrm{~Hz}), 5.26(1 \mathrm{H}, \mathrm{dddd}, J=$ $1.7,1.7,1.8,17.2 \mathrm{~Hz}), 5.11(1 \mathrm{H}, \mathrm{dddd}, J=1.5,1.5,1.8,10.5 \mathrm{~Hz}), 4.02-4.12(2 \mathrm{H}, \mathrm{m}), 3.97(1 \mathrm{H}$, dddd, $J=1.5,1.7,5.3,12.9 \mathrm{~Hz}), 3.76-3.86(2 \mathrm{H}, \mathrm{m}), 2.97(1 \mathrm{H}, \mathrm{t}, J=5.2 \mathrm{~Hz}), 2.69(1 \mathrm{H}, \mathrm{ddd}, J=$ $2.1,2.9,13.2 \mathrm{~Hz}), 2.41(1 \mathrm{H}$, dsept, $J=2.0,6.9 \mathrm{~Hz}), 1.67-1.78(1 \mathrm{H}, \mathrm{m}), 1.35-1.65(8 \mathrm{H}, \mathrm{m}), 1.16-$ $1.26(1 \mathrm{H}, \mathrm{m}), 0.91(3 \mathrm{H}, \mathrm{d}, J=6.3 \mathrm{~Hz}), 0.90(9 \mathrm{H}, \mathrm{s}), 0.89(6 \mathrm{H}, \mathrm{d}, J=6.3 \mathrm{~Hz}), 0.69(1 \mathrm{H}, \mathrm{t}, J=$ $13.2 \mathrm{~Hz})$.

${ }^{13}$ C NMR (75 MHz, $\left.\mathrm{CDCl}_{3}\right): \delta 135.8,115.6,100.7,84.3,72.2,66.7,59.2,51.4,38.8,37.4,35.8$, 35.0, 31.8, 29.0, 26.1 (3C), 24.3, 23.7, 22.2, 21.8, 18.9.

HRMS: $\left[\mathrm{M}^{+}\right]$Calculated for $\mathrm{C}_{22} \mathrm{H}_{40} \mathrm{O}_{3}: 352.2977$. Found: 352.2970.

Elemental Anal.: Calculated for $\mathrm{C}_{22} \mathrm{H}_{40} \mathrm{O}_{3}$ : C, 74.95; H, 11.44. Found: C, 75.09; H, 11.45.

$\underline{[\alpha]_{\mathrm{D}}{ }^{27}=-13.9^{\circ}\left(c 1.50, \mathrm{CH}_{2} \mathrm{Cl}_{2}\right)}$.

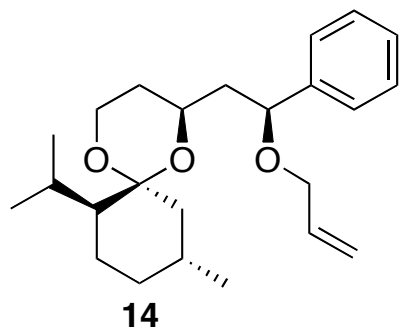


To a solution of alcohol $10(270 \mathrm{mg}, 0.812 \mathrm{mmol})$ in THF $(2.8 \mathrm{~mL})$, under $\mathrm{N}_{2}$, at $\mathrm{rt}$ was added $\mathrm{KOt}$ - $\mathrm{Bu}(227 \mathrm{mg}, 2.02 \mathrm{mmol})$. The reaction mixture was allowed to stir for $10 \mathrm{~min}$ during which time the color changed from colorless to a deep orange. Allyl bromide $(211 \mu \mathrm{L}, 2.44$ $\mathrm{mmol}$ ) was added via syringe and the reaction mixture was stirred at $\mathrm{rt}$ for $20 \mathrm{~min}$. Saturated $\mathrm{NH}_{4} \mathrm{Cl}(\mathrm{aq})(10 \mathrm{~mL})$ and brine $(30 \mathrm{~mL})$ were added and the reaction mixture was extracted $3 \times 5$ $\mathrm{mL}$ with $\mathrm{Et}_{2} \mathrm{O}$. The combined organic layers were dried with anhydrous $\mathrm{MgSO}_{4}$, filtered and concentrated in vacuo. The crude yellow oil was used without purification in the next step. An analytical sample (a clear, colorless, viscous oil) was obtained by flash chromatography of a portion of the crude material (silica; 5\% EtOAc/pentane).

Data for 14:

$\mathbf{R}_{f}=0.73$ (10\% EtOAc, 90\% pentane) .

IR (film): 3066, 3031, 2952, 2869, 1647, 1455, 1374, 1307, 1267, 1240, 1219, 1191, 1157, 1140, $1105,1022,1003 \mathrm{~cm}^{-1}$.

${ }^{1} \mathbf{H}$ NMR $\left(500 \mathrm{MHz}, \mathrm{CDCl}_{3}\right): \delta 7.32(5 \mathrm{H}, \mathrm{m}), 5.87(1 \mathrm{H}, \mathrm{dddd}, J=5.1,6.0,10.4,17.2 \mathrm{~Hz}), 5.22$ $(1 \mathrm{H}, \mathrm{qd}, J=1.7,17.2 \mathrm{~Hz}), 5.14(1 \mathrm{H}, \mathrm{tdd}, J=1.3,1.9,10.4 \mathrm{~Hz}), 4.48(1 \mathrm{H}, \mathrm{dd}, J=5.9,8.3 \mathrm{~Hz})$, $3.98(1 \mathrm{H}, \mathrm{ddd}, J=2.7,11.5,12.7 \mathrm{~Hz}), 3.85(1 \mathrm{H}, \mathrm{tdd}, J=1.5,5.1,12.8 \mathrm{~Hz}), 3.75(2 \mathrm{H}, \mathrm{m}), 3.62$ $(1 \mathrm{H}$, dddd, $J=2.6,4.2,8.4,11.2 \mathrm{~Hz}), 2.50(1 \mathrm{H}, \mathrm{ddd}, J=2.0,3.6,13.4 \mathrm{~Hz}), 2.42(1 \mathrm{H}$, dsept, $J=$ $2.0,7.0 \mathrm{~Hz}), 2.14(1 \mathrm{H}, \mathrm{ddd}, J=6.0,8.4,14.2 \mathrm{~Hz}), 1.77(1 \mathrm{H}, \mathrm{ddd}, J=4.1,8.5,13.7 \mathrm{~Hz}), 1.72$ $(1 \mathrm{H}, \mathrm{m}), 1.62(1 \mathrm{H}$, ddt, $J=5.5,11.5,12.9 \mathrm{~Hz}), 1.48(3 \mathrm{H}, \mathrm{m}), 1.32(1 \mathrm{H}, \mathrm{ddd}, J=1.6,2.7,12.9$ $\mathrm{Hz}), 1.16(1 \mathrm{H}, \mathrm{ddd}, J=2.0,4.4,12.3 \mathrm{~Hz}), 0.93(3 \mathrm{H}, \mathrm{d}, J=6.9 \mathrm{~Hz}), 0.89(3 \mathrm{H}, \mathrm{d}, J=6.9 \mathrm{~Hz}), 0.85$ $(1 \mathrm{H}, \mathrm{m}), 0.84(3 \mathrm{H}, \mathrm{d}, J=6.9 \mathrm{~Hz}), 0.63(1 \mathrm{H}, \mathrm{dd}, J=12.6,13.5 \mathrm{~Hz})$.

${ }^{13}$ C NMR (125 MHz, $\mathrm{CDCl}_{3}$ ): $\delta 141.7,134.9,128.3$ (x2), 127.8, 127.4 (x2), 116.7, 100.5, 77.7, 69.2, 64.6, 58.9, 51.2, 44.7, 37.4, 34.9, 31.6, 29.1, 24.4, 23.8, 22.0, 21.9, 19.0.

HRMS: [M+] Calculated for $\mathrm{C}_{24} \mathrm{H}_{36} \mathrm{O}_{3}: 372.2664$. Found: 372.2673 .

Elemental Anal.: Calculated for $\mathrm{C}_{24} \mathrm{H}_{36} \mathrm{O}_{3}$ : C 77.38; H 9.74. Found C 77.46; H 9.95. $[\alpha]_{D}^{25}=-26.6^{\circ}\left(c 1.32, \mathrm{CDCl}_{3}\right)$.

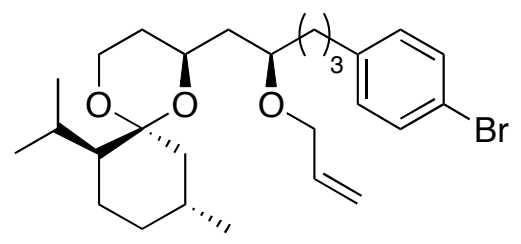

15

In a $10 \mathrm{~mL}$ round bottom flask $12(188 \mathrm{mg}, 0.33 \mathrm{mmol})$ was dissolved in THF $(2.3 \mathrm{~mL})$. The reaction flask was immersed in an ice/water bath and a $1 \mathrm{M}$ solution of TBAF in THF (993 $\mu \mathrm{L}, 0.993 \mathrm{mmol}$ ) was added in a drop-wise manner over $5 \mathrm{~min}$. The ice/water bath was removed and the reaction was stirred for $7 \mathrm{~h}$. The reaction was diluted with pentane $(40 \mathrm{~mL})$ and extracted with saturated $\mathrm{NH}_{4} \mathrm{Cl}_{\text {(aq.) }}(3 \times 40 \mathrm{~mL}), \mathrm{H}_{2} \mathrm{O}(1 \times 40 \mathrm{~mL})$, saturated $\mathrm{NaHCO}_{3 \text { (aq.) }}(2 \times 40 \mathrm{~mL})$, and 
brine (1 x $40 \mathrm{~mL}$ ). The organic phase was then dried over $\mathrm{MgSO}_{4}$, which was subsequently removed by vacuum filtration. The filtrate was then concentrated and quickly chromatographed (silica gel 3/7 $\mathrm{Et}_{2} \mathrm{O}$ :pentane). This material was re-dissolved in THF (1.14 mL). To this solution was added $t$-BuOK $(84 \mathrm{mg}, 0.745 \mathrm{mmol}$ ) and the resulting cloudy suspension was stirred for 3 min, after which time allyl bromide $(61 \mu \mathrm{L}, 0.745 \mathrm{mmol})$ was added. The resulting suspension was stirred at $\mathrm{rt}$ for $1 \mathrm{~h}$. Additional $t$-BuOK $(84 \mathrm{mg}, 0.745 \mathrm{mmol})$ and allyl bromide $(61 \mu \mathrm{L}$, $0.745 \mathrm{mmol}$ ) were added and the reaction was stirred for another hour. At this point, additional $t$-BuOK (84 mg, $0.745 \mathrm{mmol})$ and allyl bromide $(61 \mu \mathrm{L}, 0.745 \mathrm{mmol})$ were added. After another $1 \mathrm{~h}$, more $t$-BuOK $(84 \mathrm{mg}, 0.745 \mathrm{mmol})$ and allyl bromide $(61 \mu \mathrm{L}, 0.745 \mathrm{mmol})$ were added. After $1 \mathrm{~h}$ the reaction was complete; it was diluted with pentane $(1 \mathrm{x} 40 \mathrm{~mL})$ and extracted with saturated $\mathrm{NH}_{4} \mathrm{Cl}_{\text {(aq.) }}(2 \times 40 \mathrm{~mL})$, saturated $\mathrm{NaHCO}_{3 \text { (aq.) }}(2 \times 40 \mathrm{~mL})$, and brine $(1 \mathrm{x} 40 \mathrm{~mL})$. The organic phase was then dried over $\mathrm{MgSO}_{4}$, which was then removed by vacuum filtration. The filtrate was concentrated in vacuo and $\mathbf{1 5}$ was isolated from the residue by flash chromatography (silica gel 1/9 $\mathrm{Et}_{2} \mathrm{O}$ :pentane) as a clear oil (133 mg, 90\%).

Data for 15:

$\mathbf{R}_{f}=0.37\left(10 \% \mathrm{Et}_{2} \mathrm{O} /\right.$ pentane $)$.

${ }^{1} \mathbf{H}$ NMR $\left(500 \mathrm{MHz}, \mathrm{CDCl}_{3}\right): \delta 7.39(2 \mathrm{H}, \mathrm{d}, J=8 \mathrm{~Hz}), 7.05(2 \mathrm{H}, \mathrm{d}, J=8.5 \mathrm{~Hz}), 5.89(1 \mathrm{H}, \mathrm{m})$, $5.24(1 \mathrm{H}, \mathrm{dq}, J=1.5,17 \mathrm{~Hz}), 5.14(1 \mathrm{H}, \mathrm{dd}, J=1,10.5 \mathrm{~Hz}), 4.07(1 \mathrm{H}, \mathrm{td}, J=2,11.5 \mathrm{~Hz}), 3.98$ $(1 \mathrm{H}, \mathrm{ddt}, J=1.5,5.5,12.5 \mathrm{~Hz}), 3.89(1 \mathrm{H}, \mathrm{ddt}, J=1,5.5,12.5 \mathrm{~Hz}), 3.80(1 \mathrm{H}, \mathrm{ddd}, J=1.5,5,11.5$ $\mathrm{Hz}), 3.82(1 \mathrm{H}, \mathrm{m}), 3.51(1 \mathrm{H}$, sept, $J=4 \mathrm{~Hz}), 2.67(1 \mathrm{H}, \mathrm{brd}, J=13.5 \mathrm{~Hz}), 2.56(2 \mathrm{H}, \mathrm{m}), 2.41(1 \mathrm{H}$, dsept, $J=7,1.5 \mathrm{~Hz}), 1.34-1.83(12 \mathrm{H}, \mathrm{m}), 1.17(1 \mathrm{H}, \mathrm{dq}, J=2,13 \mathrm{~Hz}), 0.83-0.92(10 \mathrm{H}, \mathrm{m}), 0.68$ $(1 \mathrm{H}, \mathrm{t}, J=13 \mathrm{~Hz})$.

${ }^{13}$ C NMR (125 MHz, $\mathrm{CDCl}_{3}$ ): $\delta$ 141.4, 135.3, 131.3 (2C), 130.1 (2C), 119.4, 116.6, 100.4, 75.1, 69.5, 64.7, 59.1, 51.2, 40.6, 37.3, 35.4, 34.9, 33.5, 32.0, 29.2, 27.2, 24.3, 23.7, 22.3, 21.8, 18.9.

IR (thin film): 2949, 2867, 1488, 1456, 1374, 1306, 1266, 1142, 1118, 1072, 976, 921, $820 \mathrm{~cm}^{-1}$.

HRMS: $\left[\mathrm{M}^{+}\right]$Calculated for $\mathrm{C}_{27} \mathrm{H}_{41} \mathrm{BrO}_{3}$ : 492.2239; found: 492.2232 .

Elemental Anal: Calculated for $\mathrm{C}_{27} \mathrm{H}_{41} \mathrm{BrO}_{3}$ : C, 65.71; H, 8.37; found: C, 65.52; H, 8.56.

$[\alpha]_{\mathrm{D}}^{25}=+3.7^{\circ}\left(c 3.06, \mathrm{CH}_{2} \mathrm{Cl}_{2}\right)$.

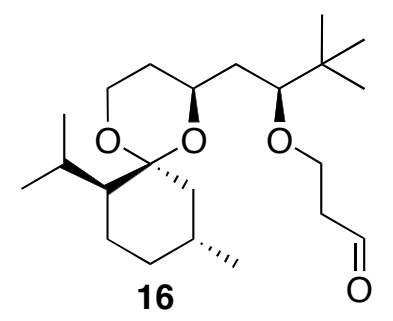

A solution of alkene $13(60.5 \mathrm{mg}, 0.172 \mathrm{mmol})$ in $0.8 \mathrm{~mL}$ THF was treated with 9-BBN $(0.5 \mathrm{M}$ in $\mathrm{THF}, 1.03 \mathrm{~mL}, 0.515 \mathrm{mmol})$ at $\mathrm{rt}$. The resulting colorless solution was stirred at $\mathrm{rt}$ for 
$40 \mathrm{~min}$ and then cooled to $5^{\circ} \mathrm{C} .3 \mathrm{M} \mathrm{NaOH}(0.85 \mathrm{~mL})$ and $30 \% \mathrm{H}_{2} \mathrm{O}_{2}(0.85 \mathrm{~mL})$ were added successively and the mixture was stirred vigorously at $\mathrm{rt}$ for $1.5 \mathrm{~h}$. Saturated $\mathrm{Na}_{2} \mathrm{SO}_{3}$ was added and the mixture was diluted with EtOAc. The organic layer was washed with brine $(2 \mathrm{x})$, dried over $\mathrm{Na}_{2} \mathrm{SO}_{4}$ and the solvent removed. The crude residue was passed through a short column of silica gel (20\% EtOAc / hexanes) to afford $65 \mathrm{mg}$ of a colorless oil.

This crude material was immediately dissolved in $3 \mathrm{~mL} \mathrm{CH} \mathrm{Cl}_{2}$ and treated with a single portion of Dess-Martin periodinane $(108 \mathrm{mg}, 0.26 \mathrm{mmol})$ at $\mathrm{rt}$. After stirring for $1 \mathrm{~h}$, the opaque white slurry was concentrated with a stream of $\mathrm{N}_{2}$ (roughly $0.5 \mathrm{~mL}$ final volume) and loaded directly onto a silica column. The product was eluted with $10 \%$ EtOAc / hexanes to afford aldehyde 16 (49.5 mg, 78\% - 2 steps) as a clear, colorless oil.

Data for 16:

$\mathbf{R}_{f}=0.49$ (15\% EtOAc / hexanes).

IR (thin film): 2953, 2870, 1729, 1456, 1383, 1307, 1266, 1158, 1137, 1107, $975 \mathrm{~cm}^{-1}$.

${ }^{1} \mathbf{H}$ NMR $\left(300 \mathrm{MHz}, \mathrm{CDCl}_{3}\right): \delta 9.77(1 \mathrm{H}, \mathrm{t}, J=2.1 \mathrm{~Hz}), 4.07(1 \mathrm{H}, \mathrm{ddd}, J=3.3,11.6,11.6 \mathrm{~Hz})$, $3.92(1 \mathrm{H}, \mathrm{dt}, J=5.7,9.6 \mathrm{~Hz}), 3.64-3.84(3 \mathrm{H}, \mathrm{m}), 2.91(1 \mathrm{H}, \mathrm{t}, J=5.0 \mathrm{~Hz}), 2.69(1 \mathrm{H}, b r \mathrm{~d}, J=$ $12.4 \mathrm{~Hz}), 2.53-2.60(2 \mathrm{H}, \mathrm{m}), 2.39(1 \mathrm{H}, \mathrm{dsept}, J=1.9,6.9 \mathrm{~Hz}), 1.33-1.76(9 \mathrm{H}, \mathrm{m}), 1.15-1.23(1 \mathrm{H}$, $\mathrm{m}), 0.89(3 \mathrm{H}, \mathrm{d}, J=6.5 \mathrm{~Hz}), 0.87(6 \mathrm{H}, \mathrm{d}, J=6.9 \mathrm{~Hz}), 0.84(9 \mathrm{H}, \mathrm{s}), 0.68(1 \mathrm{H}, \mathrm{t}, J=13.2 \mathrm{~Hz})$.

${ }^{13}$ C NMR $\left(75 \mathrm{MHz}, \mathrm{CDCl}_{3}\right): \delta 201.9,100.7,84.3,66.9,64.4,59.2,51.3,44.1,38.7,37.3,35.7$, $34.9,31.8,29.1,25.9$ (3C), 24.3, 23.7, 22.1, 21.9, 18.9 .

HRMS: $\left[\mathrm{M}^{+}\right]$Calculated for $\mathrm{C}_{22} \mathrm{H}_{40} \mathrm{O}_{4}: 368.2927$. Found: 368.2926.

Elemental Anal.: Calculated for $\mathrm{C}_{22} \mathrm{H}_{40} \mathrm{O}_{4}$ : C, 71.70; H, 10.94. Found: C, 71.48; H, 11.06.

$[\alpha]_{\mathrm{D}}^{29}-11.3^{\circ}\left(c 0.99, \mathrm{CHCl}_{3}\right)$.

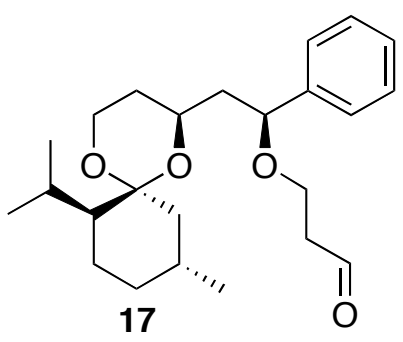

To a solution of crude allyl ether $14(232.4 \mathrm{mg})$ in THF $(2.5 \mathrm{~mL})$, under $\mathrm{N}_{2}$ at $\mathrm{rt}$, was added 9-BBN $(5.0 \mathrm{~mL}$ of a $0.5 \mathrm{M}$ solution in THF; $2.5 \mathrm{mmol})$ via syringe. The reaction was allowed to stir at $\mathrm{rt}$ for $1 \mathrm{hr}$ then the flask was immersed in a $0^{\circ} \mathrm{C}$ (external temp.) bath. Once cooled, $15 \%$ $\mathrm{NaOH}(\mathrm{aq})(3.5 \mathrm{~mL})$ and $30 \% \mathrm{H}_{2} \mathrm{O}_{2}(\mathrm{aq})(3.5 \mathrm{~mL})$ were added dropwise and the reaction mixture was stirred for $1.25 \mathrm{hr}$ at $0^{\circ} \mathrm{C}$. Saturated $\mathrm{NaS}_{2} \mathrm{O}_{3}(\mathrm{aq})(15 \mathrm{~mL})$ was added portionwise (vigorous bubbling!) followed by $50 \mathrm{~mL}$ brine. The mixture was then extracted $3 \times 50 \mathrm{~mL}$ with EtOAc. The combined organic layers were dried with anhydrous $\mathrm{MgSO}_{4}$, filtered and concentrated. Flash chromatography of the resulting oil on silica (30\% EtOAc/pentane) gave $253 \mathrm{mg}$ of an 
inseparable mixture of materials as a pale yellow oil ( $\mathrm{Rf}=0.54$ in $30 \%$ EtOAc/pentane). This mixture was used directly in the next step.

To a solution of the crude mixture in $\mathrm{CH}_{2} \mathrm{Cl}_{2}(16 \mathrm{~mL})$, under $\mathrm{N}_{2}$, at $\mathrm{rt}$ was added Dess-Martin periodinane $(550 \mathrm{mg} ; 1.30 \mathrm{mmol})$. The reaction mixture was allowed to stir for $1 \mathrm{hr}$ then sat. $\mathrm{NaHCO}_{3}(\mathrm{aq})(8 \mathrm{~mL})$ and sat. $\mathrm{Na}_{2} \mathrm{~S}_{2} \mathrm{O}_{3}(\mathrm{aq})(8 \mathrm{~mL})$ were added. After $5 \mathrm{~min}$ of stirring, the reaction mixture was diluted with sat. $\mathrm{Na}_{2} \mathrm{~S}_{2} \mathrm{O}_{3}(\mathrm{aq})(20 \mathrm{~mL})$ and extracted $3 \times 75 \mathrm{~mL}$ with $\mathrm{Et}_{2} \mathrm{O}$. The combined organic layers were dried with $\mathrm{Na}_{2} \mathrm{SO}_{4}$, filtered and concentrated. Flash chromatography of the resulting oil on silica (10\% EtOAc/pentane) gave $165.4 \mathrm{mg}$ of aldehyde 17 as a clear colorless oil (67\% over 3 steps).

Data for 17:

$\mathbf{R}_{f}=0.52(15 \%$ EtOAc, $85 \%$ pentane $)$.

IR (thin film): 3065, 3030, 2952, 2869, 2725, 1727, 1454, 1376, 1307, 1265, 1157, 1140, 1101 $\mathrm{cm}^{-1}$.

${ }^{1} \mathbf{H}$ NMR $\left(500 \mathrm{MHz}, \mathrm{CDCl}_{3}\right): \delta 9.72(1 \mathrm{H}, \mathrm{t}, J=2.0 \mathrm{~Hz}), 7.33(5 \mathrm{H}, \mathrm{m}), 4.41(1 \mathrm{H}, \mathrm{dd}, J=6.0,8.3$ $\mathrm{Hz}), 3.97(1 \mathrm{H}, \mathrm{ddd}, J=2.7,11.7,12.8 \mathrm{~Hz}), 3.75(1 \mathrm{H}, \mathrm{ddd}, J=1.8,5.4,11.5 \mathrm{~Hz}), 3.60(3 \mathrm{H}, \mathrm{m})$, $2.58(2 \mathrm{H}, \mathrm{dt}, J=2.0,6.0 \mathrm{~Hz}), 2.49(1 \mathrm{H}, \mathrm{ddd}, J=2.2,3.7,13.5 \mathrm{~Hz}), 2.42(1 \mathrm{H}$, dsept, $J=2.0,7.1$ $\mathrm{Hz}), 2.09(1 \mathrm{H}, \mathrm{ddd}, J=5.9,8.3,13.9 \mathrm{~Hz}), 1.73(2 \mathrm{H}, \mathrm{m}), 1.61(1 \mathrm{H}, \mathrm{ddt}, J=5.4,11.5,12.9 \mathrm{~Hz})$, $1.48(3 \mathrm{H}, \mathrm{m}), 1.31(1 \mathrm{H}, \mathrm{ddt}, J=1.7,12.9,2.7 \mathrm{~Hz}), 1.17(1 \mathrm{H}, \mathrm{ddd}, J=2.0,4.2,12.5 \mathrm{~Hz}), 0.93$ $(3 \mathrm{H}, \mathrm{d}, J=7.0 \mathrm{~Hz}), 0.90(3 \mathrm{H}, \mathrm{d}, J=7.0 \mathrm{~Hz}), 0.87(1 \mathrm{H}, \mathrm{m}), 0.84(3 \mathrm{H}, \mathrm{d}, J=6.7 \mathrm{~Hz}), 0.63(1 \mathrm{H}$, $\mathrm{dd}, J=12.4,13.5 \mathrm{~Hz})$.

${ }^{13}$ C NMR (75 MHz, $\left.\mathrm{CDCl}_{3}\right): \delta$ 201.4, 141.4, 128.5 (x2), 128.0, 127.2 (x2), 100.5, 79.1, 64.5, 62.1, 58.9, 51.2, 44.7, 43.8, 37.3, 34.8, 31.5, 29.1, 24.3, 23.8, 22.0 (x2), 19.0.

HRMS: $\left[\mathrm{M}^{+}\right]$Calculated for $\mathrm{C}_{24} \mathrm{H}_{36} \mathrm{O}_{4}$ : 388.2614. Found 388.2622.

Elemental Anal.: Calculated for $\mathrm{C}_{24} \mathrm{H}_{36} \mathrm{O}_{4}$ : C 74.19; H 9.34. Found C 73.91; H 9.58.

$[\alpha]_{D}^{25}=-20.8^{\circ}\left(c 1.77, \mathrm{CH}_{2} \mathrm{Cl}_{2}\right)$.

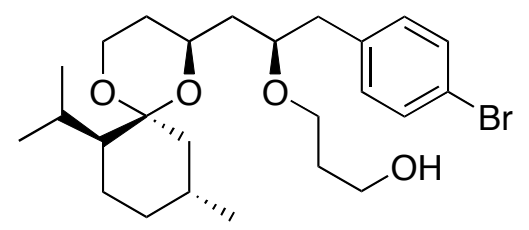

III

In a $10 \mathrm{~mL}$ round bottom flask $15(133 \mathrm{mg}, 0.27 \mathrm{mmol})$ was dissolved in a $1 \mathrm{M}$ solution of 9-BBN in THF (2.7 mL, $1.35 \mathrm{mmol})$. This solution was stirred for $4.5 \mathrm{~h}$ and then the reaction flask was immersed in an ice/water bath. A $1 \mathrm{~N}$ solution of $\mathrm{NaOH}_{\text {(aq.) }}(1.35 \mathrm{~mL})$ was added to the reaction followed by a $30 \% \mathrm{w} / \mathrm{w}$ solution of $\mathrm{H}_{2} \mathrm{O}_{2 \text { (aq.) }}(1.35 \mathrm{~mL})$. The reaction was removed from the ice/water bath and left to stir for $2 \mathrm{~h}$. Excess $\mathrm{H}_{2} \mathrm{O}_{2}$ was quenched by the drop-wise addition of a saturated solution of $\mathrm{Na}_{2} \mathrm{~S}_{2} \mathrm{O}_{3 \text { (aq.) }}$. The organic phase was then diluted with pentane $(30 \mathrm{~mL})$ and extracted with brine $(2 \times 30 \mathrm{~mL})$. The organic phase was then dried over $\mathrm{MgSO}_{4}$, which was 
subsequently removed by vacuum filtration. The filtrate was concentrated in vacuo and III was isolated from the residue by flash chromatography (silica gel 1/3 EtOAc:pentane) as a clear oil (102 mg, 74\%).

Data for III:

$\mathbf{R}_{f}=0.26(25 \%$ EtOAc / pentane $)$.

${ }^{1} \mathbf{H}$ NMR $\left(500 \mathrm{MHz}, \mathrm{CDCl}_{3}\right): \delta 7.39(2 \mathrm{H}, \mathrm{d}, J=8 \mathrm{~Hz}), 7.05(2 \mathrm{H}, \mathrm{d}, J=8.5 \mathrm{~Hz}), 4.07(1 \mathrm{H}, \mathrm{td}, J=$ $2.5,12.5 \mathrm{~Hz}), 3.80(2 \mathrm{H}, \mathrm{m}), 3.76(2 \mathrm{H}, \mathrm{q}, J=5.5 \mathrm{~Hz}), 3.64(1 \mathrm{H}, \mathrm{ddd}, J=5,6.5,9 \mathrm{~Hz}), 3.52(1 \mathrm{H}$, $\mathrm{ddd}, J=5,6.59 \mathrm{~Hz}), 3.45(1 \mathrm{H}$, sept, $J=3.5 \mathrm{~Hz}), 2.66(1 \mathrm{H}, \mathrm{dq}, J=1.5,13.5 \mathrm{~Hz}), 2.56(2 \mathrm{H}, \mathrm{m})$, $2.43(1 \mathrm{H}, \mathrm{t}, J=5.5 \mathrm{~Hz}), 2.40(1 \mathrm{H}$, dsept, $J=7,1.5 \mathrm{~Hz}), 1.32-1.86(14 \mathrm{H}, \mathrm{m}), 1.17(1 \mathrm{H}, \mathrm{dq}, J=2$, $13 \mathrm{~Hz}), 0.83-0.91(10 \mathrm{H}, \mathrm{m}), 0.69(1 \mathrm{H}, \mathrm{t}, J=13 \mathrm{~Hz})$.

${ }^{13}$ C NMR (125 MHz, $\mathrm{CDCl}_{3}$ ): $\delta$ 141.2, 131.4 (2 C), 130.1 (2 C), 119.5, 100.4, 76.4, 68.2, 64.6, $62.4,59.1,51.2$, 40.4, 37.3, 35.4, 34.9, 33.5, 32.2, 32.0, 29.3, 27.1, 24.3, 23.7, 22.2, 21.9, 18.9 .

IR (thin film): 3442, 2948, 2867, 1488, 1456, 1374, 1307, 1266, 1142, 1111, 1072, 1011, 975, $921,797 \mathrm{~cm}^{-1}$.

HRMS: $\left[\mathrm{M}^{+}\right]$Calculated for $\mathrm{C}_{27} \mathrm{H}_{43} \mathrm{BrO}_{4}$ : 510.2345; found: 510.2364.

$[\alpha]_{\mathrm{D}}^{22}=+7.0^{\circ}\left(c 1.73, \mathrm{CH}_{2} \mathrm{Cl}_{2}\right)$.

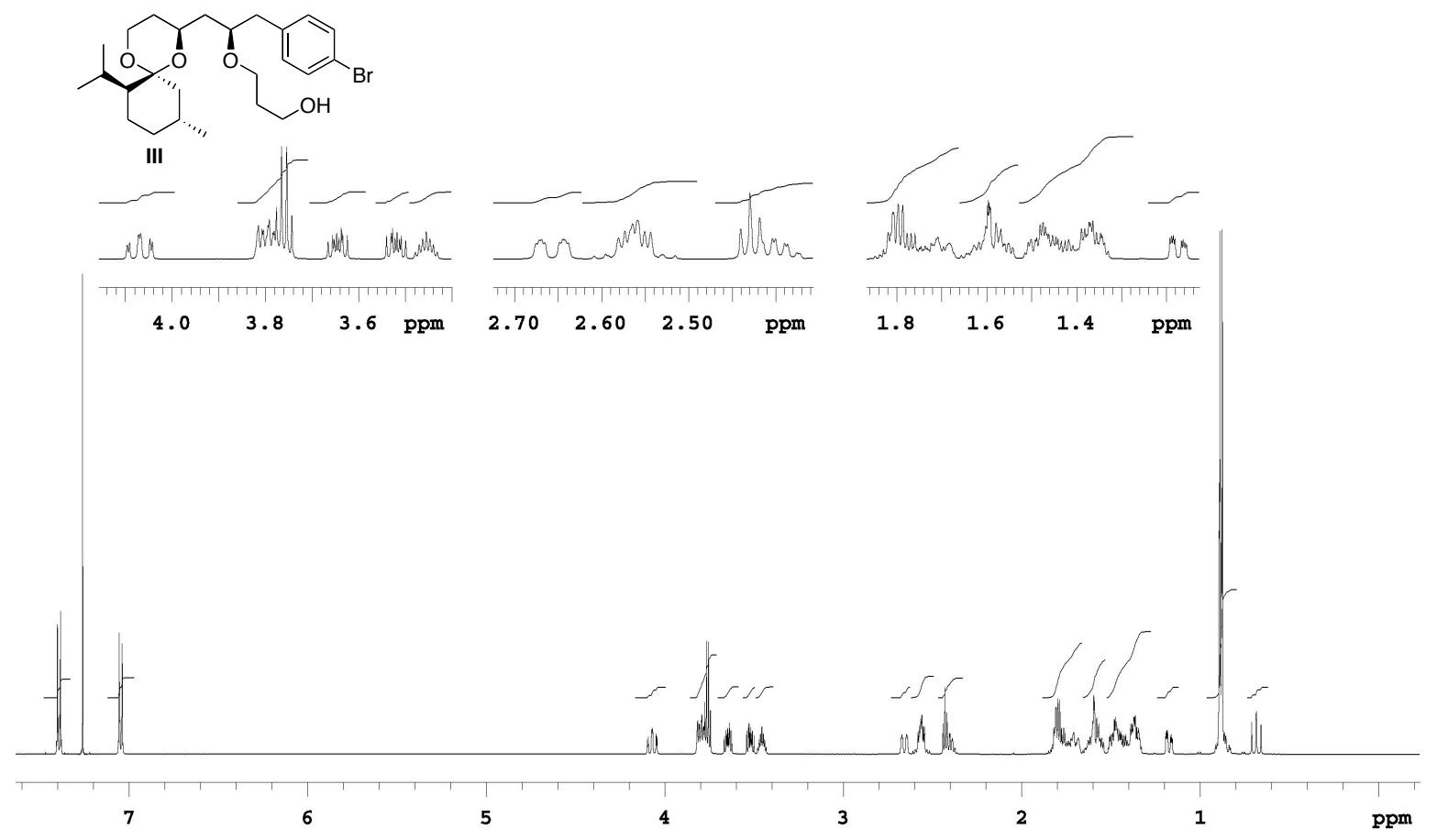




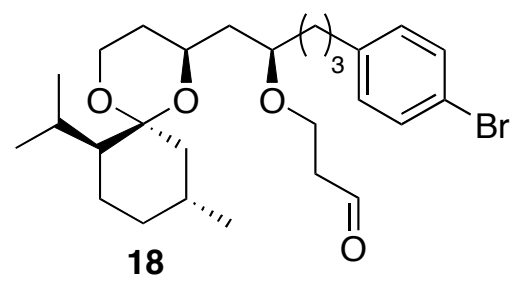

In a $10 \mathrm{~mL}$ round bottom flask III $(49 \mathrm{mg}, 0.096 \mathrm{mmol})$ was dissolved in $\mathrm{CH}_{2} \mathrm{Cl}_{2}(3.31$ $\mathrm{mL})$. To this solution was added Dess-Martin periodinane $(61 \mathrm{mg}, 0.144 \mathrm{mmol})$ in one portion. The reaction was stirred for $1.5 \mathrm{~h}$ at $\mathrm{rt}$. The reaction was then diluted with $\mathrm{Et}_{2} \mathrm{O}(30 \mathrm{~mL})$ and this solution was extracted with a 1:1 solution of saturated $\mathrm{NaHCO}_{3 \text { (aq.) }}$ : saturated $\mathrm{Na}_{2} \mathrm{SO}_{3 \text { (aq.) }}(3 \times 40$ $\mathrm{mL})$ followed by brine $(1 \times 40 \mathrm{~mL})$. The organic phase was dried over $\mathrm{MgSO}_{4}$, which was removed by vacuum filtration. The filtrate was subsequently concentrated in vacuo and $\mathbf{1 8}$ was isolated from this residue by flash chromatography (silica gel 3/17 EtOAc:pentane) as a clear oil (44 mg, 90\%).

Data for 18:

$\mathbf{R}_{f}=0.39(15 \%$ EtOAc / pentane $)$.

${ }^{1} \mathbf{H}$ NMR $\left(500 \mathrm{MHz}, \mathrm{CDCl}_{3}\right): \delta 9.78(1 \mathrm{H}, \mathrm{t}, J=2 \mathrm{~Hz}), 7.39(2 \mathrm{H}, \mathrm{d}, J=8.5 \mathrm{~Hz}), 7.04(2 \mathrm{H}, \mathrm{d}, J=$ $8.5 \mathrm{~Hz}), 4.07(1 \mathrm{H}, \mathrm{td}, J=2.5,12.5 \mathrm{~Hz}), 3.79(3 \mathrm{H}, \mathrm{m}), 3.66(1 \mathrm{H}, \mathrm{ddd}, J=5.6,6,9.5 \mathrm{~Hz}), 3.47$ $(1 \mathrm{H}$, sept, $J=4 \mathrm{~Hz}), 2.66(1 \mathrm{H}$, brd, $J=13 \mathrm{~Hz}), 2.62(2 \mathrm{H}, \mathrm{m}), 2.55(2 \mathrm{H}, \mathrm{m}), 2.40(1 \mathrm{H}$, dsept,$J=$ 7, $1.5 \mathrm{~Hz}), 1.32-1.81(12 \mathrm{H}, \mathrm{m}), 1.70(1 \mathrm{H}, \mathrm{dq}, J=2,13 \mathrm{~Hz}), 0.82-0.91(10 \mathrm{H}, \mathrm{m}), 0.68(1 \mathrm{H}, \mathrm{t}, J=$ $13 \mathrm{~Hz})$.

${ }^{13}$ C NMR (125 MHz, $\left.\mathrm{CDCl}_{3}\right): \delta 201.3,141.3,131.3$ (2 C), 130.1 (2C), 119.4, 100.4, 76.2, 64.6, 62.2, 59.0, 51.1, 44.0, 40.4, 37.3, 35.4, 34.9, 33.4, 31.9, 29.2, 27.1, 24.2, 23.7, 22.2, 21.8, 18.8 .

IR (thin film): 2949, 2867, 2723, 1728, 1488, 1456, 1374, 1306, 1266, 1218, 1156, 1142, 1111 , $1072,1011,976,958,921,831,799 \mathrm{~cm}^{-1}$.

Elemental Anal.: Calculated for $\mathrm{C}_{27} \mathrm{H}_{41} \mathrm{BrO}_{4}$ : C, 63.65; H, 8.11; found: $\mathrm{C}, 63.50 ; \mathrm{H}, 8.16$.

HRMS: [M+] Calculated for $\mathrm{C}_{27} \mathrm{H}_{41} \mathrm{BrO}_{4}$ : 508.2188; found: 508.2194.

$[\alpha]_{\mathrm{D}}^{23}=+10.3^{\circ}\left(c 2.93, \mathrm{CH}_{2} \mathrm{Cl}_{2}\right)$.

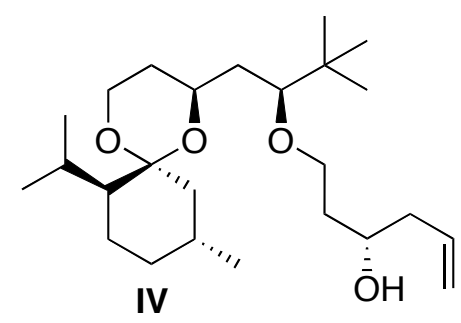

To a solution of (-)-Ipc ${ }_{2} \mathrm{BOMe}(55.0 \mathrm{mg}, 0.174 \mathrm{mmol}$, weighed under an inert atmosphere) in $\mathrm{Et}_{2} \mathrm{O}(1.0 \mathrm{~mL})$ under $\mathrm{N}_{2}$ at $-78^{\circ} \mathrm{C}$ (external temp.), was added allylmagnesium bromide $(0.8 \mathrm{M}$ in 
$\mathrm{Et}_{2} \mathrm{O}, 0.15 \mathrm{~mL}, 0.186 \mathrm{mmol}$ ) via syringe. The solution was slowly warmed to $\mathrm{rt}$ and stirred for 50 $\mathrm{min}$; a white precipitate formed after the first $10 \mathrm{~min}$ of stirring. The flask was cooled back to $-78^{\circ} \mathrm{C}$ (external temp.) and aldehyde $\mathbf{1 6}(32.0 \mathrm{mg}, 0.0868 \mathrm{mmol})$, as a solution in $1.0 \mathrm{~mL} \mathrm{Et}_{2} \mathrm{O}$, was added drop-wise to the mixture. The suspension was stirred for $1.25 \mathrm{hr}$ and then the temperature slowly warmed to rt over $30 \mathrm{~min} . \mathrm{H}_{2} \mathrm{O}_{2}(0.2 \mathrm{~mL}$ of a $30 \%$ aq. solution $)$ and $\mathrm{NaOH}$ $(0.6 \mathrm{~mL}$ of a $15 \%$ aq. solution) were added and the biphasic mixture was allowed to stir for 45 min. The mixture was diluted with brine and extracted with EtOAc $(3 \times 25 \mathrm{~mL})$. The combined organic layers were dried over anhydrous $\mathrm{Na}_{2} \mathrm{SO}_{4}$, filtered and concentrated in vacuo. The resulting oil was chromatographed on silica (10\% EtOAc / pentane) to give $28.5 \mathrm{mg}$ of the homo-allylic alcohol IV as a colorless oil (80\%).

Data for IV:

$\mathbf{R}_{f}=0.25$ (10\% EtOAc, $90 \%$ pentane).

IR (thin film): 3470, 3077, 2952, 2869, 1641, 1456, 1362, 1307, 1265, 1218, 1158, 1138, 1107 $\mathrm{cm}^{-1}$.

${ }^{1} \mathbf{H}$ NMR $\left(500 \mathrm{MHz}, \mathrm{CDCl}_{3}\right): \delta 5.84(1 \mathrm{H}, \mathrm{ddt}, J=10.3,17.2,7.1 \mathrm{~Hz}), 5.10(2 \mathrm{H}, \mathrm{m}), 4.07(1 \mathrm{H}$, ddd, $J=1.5,5.2,11.7 \mathrm{~Hz}), 3.88(2 \mathrm{H}, \mathrm{m}), 3.83(1 \mathrm{H}, \mathrm{ddd}, J=1.5,5.2,11.7 \mathrm{~Hz}), 3.77(1 \mathrm{H}, \mathrm{m})$, $3.56(1 \mathrm{H}$, ddd, $J=4.2,8.9,9.7 \mathrm{~Hz}), 3.39(1 \mathrm{H}, \mathrm{d}, J=2.4 \mathrm{~Hz}), 2.94(1 \mathrm{H}, \mathrm{t}, J=5.1 \mathrm{~Hz}), 2.70(1 \mathrm{H}$, ddd, $J=1.9,3.3,13.5 \mathrm{~Hz}), 2.41(1 \mathrm{H}$, dsept, $J=2.3,7.0 \mathrm{~Hz}), 2.25(2 \mathrm{H}, \mathrm{m}), 1.40-1.80(10 \mathrm{H}, \mathrm{m})$, $1.20(1 \mathrm{H}, \mathrm{ddd}, J=2.2,3.9,12.6 \mathrm{~Hz}), 0.90(10 \mathrm{H}, \mathrm{m}), 0.89(9 \mathrm{H}, \mathrm{s}), 0.70(1 \mathrm{H}, \mathrm{dd}, J=12.5,13.6$ $\mathrm{Hz})$.

${ }^{13}$ C NMR $\left(125 \mathrm{MHz}, \mathrm{CDCl}_{3}\right): \delta 135.0,117.3,100.7,84.8,71.3,70.2,66.8,59.2,51.4,41.9$, 38.9, 37.4, 36.1, 35.7, 35.0, 31.9, 29.1, 26.1 (x3), 24.3, 23.8, 22.3, 21.9, 19.0.

HRMS: $\left[\mathrm{M}^{+}\right]$Calculated for $\mathrm{C}_{25} \mathrm{H}_{46} \mathrm{O}_{4}: 410.3396$. Found: 410.3399.

$[\alpha]_{D}^{26}=-11.0^{\circ}\left(c 0.71, \mathrm{CDCl}_{3}\right)$. 

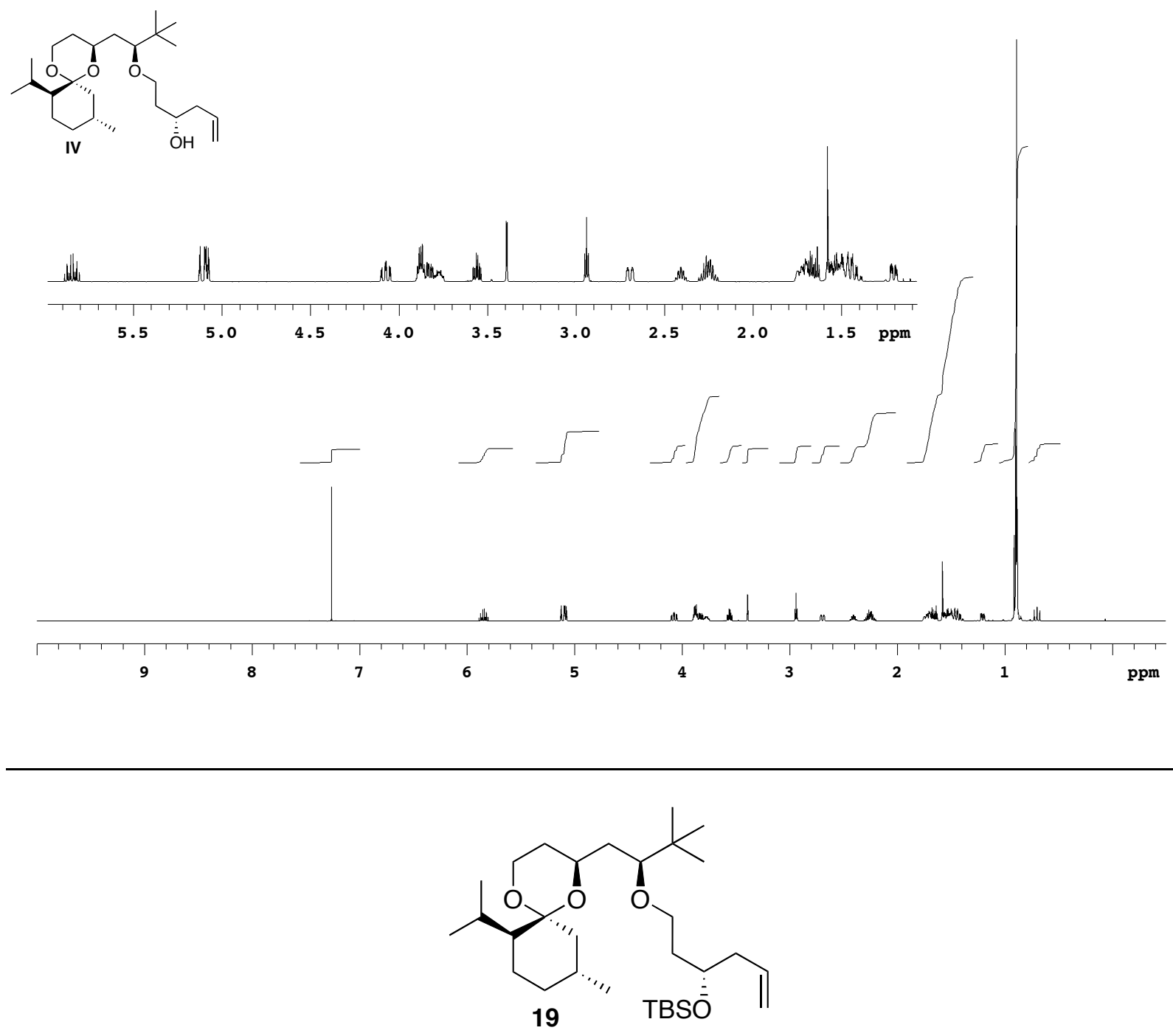

To a solution of homo-allylic alcohol $\mathbf{I}(76.0 \mathrm{mg}, 0.185 \mathrm{mmol})$ in $\mathrm{CH}_{2} \mathrm{Cl}_{2}(1.0 \mathrm{~mL})$, under $\mathrm{N}_{2}$ at $\mathrm{rt}$, was added imidazole $(83.8 \mathrm{mg}, 0.556 \mathrm{mmol})$ and $\mathrm{TBSCl}(72.0 \mathrm{mg}, 1.11 \mathrm{mmol})$. The mixture was allowed to stir for $20 \mathrm{hr}$ and then diluted with sat. aq. $\mathrm{NaHCO}_{3}$ and extracted with EtOAc $(3 \times 25 \mathrm{~mL})$. The combined organic layers were dried over anhydrous $\mathrm{Na}_{2} \mathrm{SO}_{4}$, filtered and concentrated in vacuo. Chromatography on silica (4\% EtOAc / pentane) gave $81.8 \mathrm{mg}$ of the silyl ether 19 as a clear colorless oil (84\%).

Data for 19:

$\mathbf{R}_{f}=0.69$ (5\% EtOAc, $95 \%$ pentane $)$.

IR (thin film): 3077, 2953, 2868, 1642, 1471, 1362, 1307, 1256, 1218, 1157, 1137, 1109, 1004, $976,912,837,775 \mathrm{~cm}^{-1}$.

${ }^{1} \mathbf{H}$ NMR (500 MHz, $\left.\mathrm{C}_{6} \mathrm{D}_{6}\right): \delta 5.92(1 \mathrm{H}, \mathrm{ddt}, J=10.1,17.1,7.2 \mathrm{~Hz}), 5.09(2 \mathrm{H}, \mathrm{m}), 3.96(1 \mathrm{H}$, quint, $J=5.8 \mathrm{~Hz}), 3.87(1 \mathrm{H}$, ddd, $J=2.6,11.6,12.6 \mathrm{~Hz}), 3.77(2 \mathrm{H}, \mathrm{m}), 3.69(1 \mathrm{H}, \mathrm{ddd}, J=1.4$, $5.3,11.5 \mathrm{~Hz}), 3.51(1 \mathrm{H}, \mathrm{dt}, J=6.5,9.5 \mathrm{~Hz}), 2.95(1 \mathrm{H}, \mathrm{t}, J=5.5 \mathrm{~Hz}), 2.83(1 \mathrm{H}, \mathrm{dsept}, J=2.0,7.0$ 
$\mathrm{Hz}), 2.69(1 \mathrm{H}, \mathrm{ddd}, J=2.1,3.4,13.4 \mathrm{~Hz}), 2.31(2 \mathrm{H}, \mathrm{m}), 1.50-1.90(8 \mathrm{H}, \mathrm{m}), 1.42(1 \mathrm{H}$, ddt, $J=$ 5.4, 11.6, $12.8 \mathrm{~Hz}), 1.39(1 \mathrm{H}, \mathrm{m}), 1.18(3 \mathrm{H}, \mathrm{d}, J=7 \mathrm{~Hz}), 1.08(3 \mathrm{H}, \mathrm{d}, J=7.0 \mathrm{~Hz}), 1.00(9 \mathrm{H}, \mathrm{s}, \mathrm{t}-$ $\mathrm{Bu}), 1.00(1 \mathrm{H}, \mathrm{m}), 0.94(9 \mathrm{H}, \mathrm{s}), 0.91(3 \mathrm{H}, \mathrm{d}, J=7.0 \mathrm{~Hz}), 0.84(1 \mathrm{H}, \mathrm{dq}, J=4.0,12.5 \mathrm{~Hz}), 0.72$ $(1 \mathrm{H}, \mathrm{dd}, J=12.4,13.4 \mathrm{~Hz}), 0.13(3 \mathrm{H}, \mathrm{s}), 0.09(3 \mathrm{H}, \mathrm{s})$.

${ }^{13}$ C NMR $\left(125 \mathrm{MHz}, \mathrm{C}_{6} \mathrm{D}_{6}\right): \delta 135.5,117.0,100.8,84.2,69.8,67.7,67.2,59.2,51.8,42.4,39.2$, 37.8, 37.7, 35.9, 35.4, 32.2, 29.4, 26.4 (x3), 26.1 (x3), 24.8, 24.2, 22.5 (x2), 19.6, 18.3, -4.2, -4.4;

HRMS: $\left[\mathrm{M}^{+}\right]$Calculated for $\mathrm{C}_{31} \mathrm{H}_{60} \mathrm{O}_{4} \mathrm{Si}$ : 524.4261. Found: 524.4252.

$[\alpha]_{D}^{25}=+4.3^{\circ}\left(c 0.70, \mathrm{CH}_{2} \mathrm{Cl}_{2}\right)$
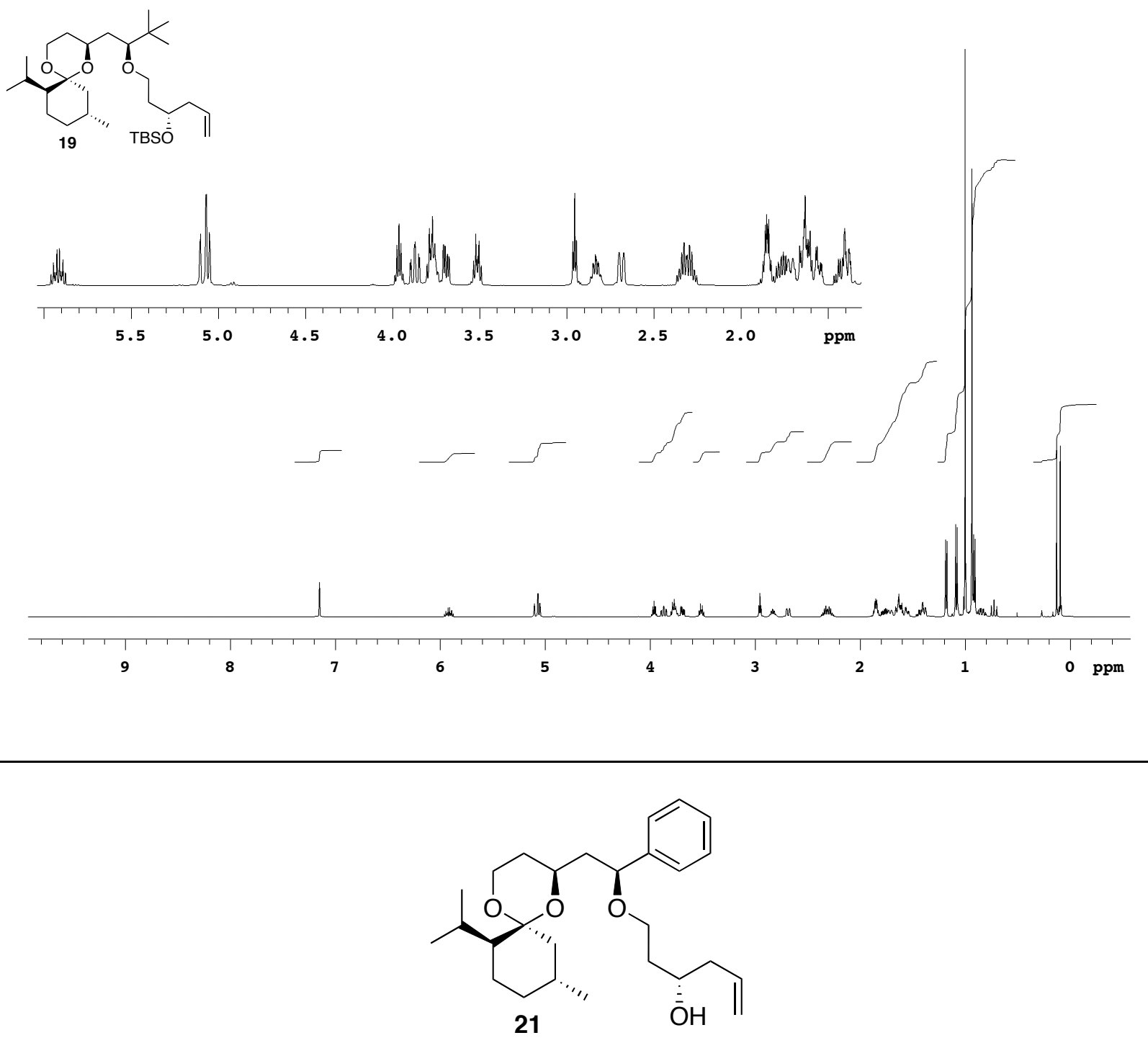

To a solution of (-)- $\mathrm{Ipc}_{2} \mathrm{BOMe}(87.0 \mathrm{mg}, 0.275 \mathrm{mmol}$, weighed under an inert atmosphere $)$ in $\mathrm{Et}_{2} \mathrm{O}(1.8 \mathrm{~mL})$ under $\mathrm{N}_{2}$ at $-78^{\circ} \mathrm{C}$ (external temp.), was added allylmagnesium bromide $(0.8 \mathrm{M}$ in $\left.\mathrm{Et}_{2} \mathrm{O}, 0.30 \mathrm{~mL}, 0.24 \mathrm{mmol}\right)$ via syringe. The bath was removed and the solution stirred for $1 \mathrm{hr}$ 
while it warmed to rt; a white precipitate formed after the first $10 \mathrm{~min}$ of stirring. The flask was cooled back to $-78^{\circ} \mathrm{C}$ (external temp.) and aldehyde $17(53.1 \mathrm{mg}, 0.137 \mathrm{mmol})$, as a solution in $1.8 \mathrm{~mL} \mathrm{Et}_{2} \mathrm{O}$, was added drop-wise to the mixture over $1 \mathrm{~min}$. The suspension was stirred for 1.5 $\mathrm{hr}$ and then the temperature slowly warmed to rt over $30 \mathrm{~min} . \mathrm{H}_{2} \mathrm{O}_{2}(1.1 \mathrm{~mL}$ of a $30 \%$ aq. solution) and $\mathrm{NaOH}(1.1 \mathrm{~mL}$ of a $15 \%$ aq. solution) were added and the biphasic mixture was allowed to gently reflux in a warm water bath for $1.5 \mathrm{hr} . \mathrm{Na}_{2} \mathrm{~S}_{2} \mathrm{O}_{3}(2.0 \mathrm{~mL}$ of a sat. aq. solution) was added slowly (vigorous bubbling!). The mixture was diluted with brine $(60 \mathrm{~mL})$ and extracted with EtOAc $(3 \times 25 \mathrm{~mL})$. The combined organic layers were dried over anhydrous $\mathrm{MgSO}_{4}$, filtered and concentrated in vacuo. The resulting oil was chromatographed on silica (10\% EtOAc / pentane) to give $88.7 \mathrm{mg}$ of a mixture of the two diastereomeric alcohols and pinanol as a pale pink oil.

To a solution of the crude mixture in $\mathrm{CH}_{2} \mathrm{Cl}_{2}(2.7 \mathrm{~mL})$, under $\mathrm{N}_{2}$ at $\mathrm{rt}$, was added imidazole (186 mg, $2.73 \mathrm{mmol}$ ) and TBSCl (207 mg, $1.37 \mathrm{mmol})$. The mixture was allowed to stir for 18 hr and then diluted with $\mathrm{NaHCO}_{3}\left(3 \mathrm{~mL}\right.$ of a sat. aq. solution) and extracted with $\mathrm{Et}_{2} \mathrm{O}(4 \times 3 \mathrm{~mL})$. The combined organic layers were dried over anhydrous $\mathrm{MgSO}_{4}$, filtered and concentrated in vacuo. Chromatography on silica (2\% EtOAc / pentane) gave $53.4 \mathrm{mg}$ of an inseparable mixture of the two diastereomeric silyl ethers $(\mathbf{2 0})$ as a clear colorless oil.

A portion of the diastereomeric mixture $(37.4 \mathrm{mg}, 0.0686 \mathrm{mmol})$ was dissolved in TBAF $\left(0.69 \mathrm{~mL}\right.$ of a $1.0 \mathrm{M}$ solution in THF) and allowed to stir at $\mathrm{rt}$ for $2 \mathrm{hr} 40 \mathrm{~min}$. $\mathrm{NH}_{4} \mathrm{Cl}(2 \mathrm{~mL}$ of a sat. aq. solution) was added and the mixture was extracted with EtOAc $(3 \times 3 \mathrm{~mL})$. The combined organic layers were dried over anhydrous $\mathrm{MgSO}_{4}$, filtered and concentrated in vacuo. Repeated chromatography on silica $(10 \rightarrow 15 \%$ EtOAc / pentane) allowed for the separation of the two diastereomers giving $13.0 \mathrm{mg}$ of diastereomerically pure 21 and $13.5 \mathrm{mg}$ of a diastereomeric mixture (32\% yield for the pure diastereomer over 3 steps).

Data for 21:

$\mathbf{R}_{f}=0.49(15 \%$ EtOAc $85 \%$ pentane $)$.

IR (thin film): 3467(br), 3068, 3032, 2950, 2868, 1638, 1454, 1375, 1307, 1266, 1240, 1219, $1191,1157,1140,1099 \mathrm{~cm}^{-1}$.

${ }^{1} \mathbf{H}$ NMR $\left(500 \mathrm{MHz}, \mathrm{CDCl}_{3}\right): \delta 7.32(5 \mathrm{H}, \mathrm{m}), 5.81(1 \mathrm{H}, \mathrm{ddt}, J=10.8,16.2,7.2 \mathrm{~Hz}), 5.09(2 \mathrm{H}$, $\mathrm{m}), 4.40(1 \mathrm{H}, \mathrm{dd}, J=5.7,8.6 \mathrm{~Hz}), 3.96(1 \mathrm{H}, \mathrm{ddd}, J=2.6,11.7,12.5 \mathrm{~Hz}), 3.76(2 \mathrm{H}, \mathrm{m}), 3.55(2 \mathrm{H}$, $\mathrm{m}), 3.40(1 \mathrm{H}, \mathrm{ddd}, J=4.5,8.5,9.3 \mathrm{~Hz}), 3.06(1 \mathrm{H}, \mathrm{d}, J=2.7 \mathrm{~Hz}), 2.48(1 \mathrm{H}, \mathrm{ddd}, J=1.8,3.5$, $13.4 \mathrm{~Hz}), 2.42(1 \mathrm{H}$, dsept, $J=1.6,7.0 \mathrm{~Hz}), 2.21(2 \mathrm{H}, \mathrm{m}), 2.12(1 \mathrm{H}, \mathrm{ddd}, J=5.7,8.6,13.8 \mathrm{~Hz})$, 1.38 - $1.80(8 \mathrm{H}, \mathrm{m}, \mathrm{C} 2, \mathrm{C} 4, \mathrm{C} 8, \mathrm{C} 9), 1.30(1 \mathrm{H}, \mathrm{ddt}, J=1.6,12.9,2.6 \mathrm{~Hz}), 1.16(1 \mathrm{H}, \mathrm{ddd}, J=2.0$, $4.5,12.2 \mathrm{~Hz}), 0.93(3 \mathrm{H}, \mathrm{d}, J=7.0 \mathrm{~Hz}), 0.90(3 \mathrm{H}, \mathrm{d}, J=7.0 \mathrm{~Hz}), 0.86(1 \mathrm{H}, \mathrm{m}), 0.83(3 \mathrm{H}, \mathrm{d}, J=$ $6.8 \mathrm{~Hz}), 0.63(1 \mathrm{H}, \mathrm{dd}, J=12.6,13.7 \mathrm{~Hz})$.

${ }^{13}$ C NMR (125 MHz, $\mathrm{CDCl}_{3}$ ): $\delta$ 141.3, 134.9, 128.5 (x2), 128.0, 127.3 (x2), 117.4, 100.5, 79.4, 70.7, 67.4, 64.5, 58.9, 51.2, 44.6, 41.8, 37.4, 35.8, 34.8, 31.6, 29.1, 24.4, 23.8, 21.98, 21.97, 19.0.

HRMS: $\left[\mathrm{M}^{+}\right]$Calculated for $\mathrm{C}_{27} \mathrm{H}_{42} \mathrm{O}_{4}: 430.3083$. Found: 430.3089 .

$[\alpha]_{D}^{23}=-18.2^{\circ}\left(c 0.99, \mathrm{CHCl}_{3}\right)$ 

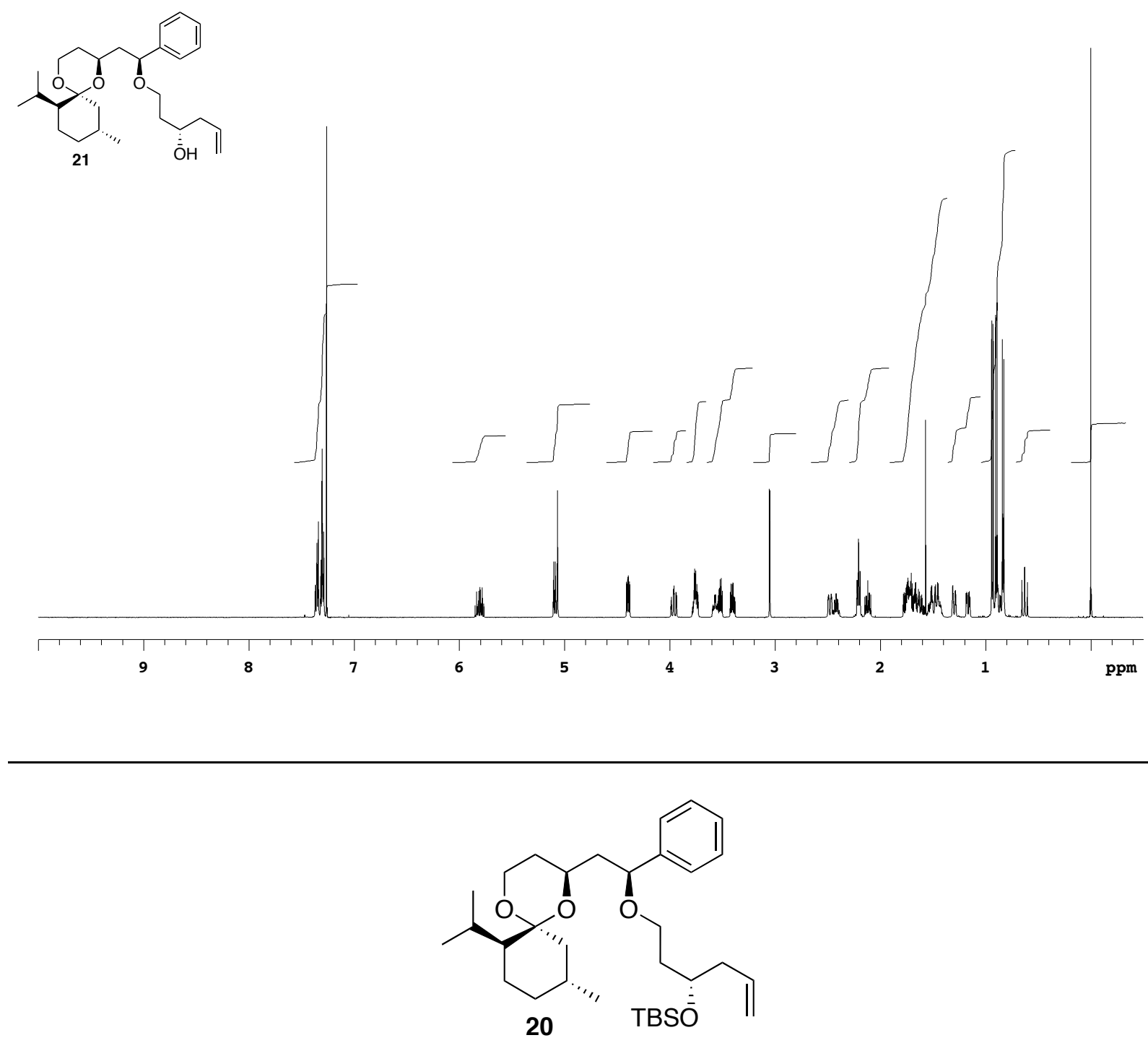

To a solution of the diastereomerically pure alcohol $21(15.9 \mathrm{mg}, 0.0369 \mathrm{mmol})$ in $\mathrm{CH}_{2} \mathrm{Cl}_{2}$ $(0.5 \mathrm{~mL})$, under $\mathrm{N}_{2}$ at $\mathrm{rt}$, was added imidazole $(5.0 \mathrm{mg}, 0.073 \mathrm{mmol})$ and $\mathrm{TBSCl}(8.4 \mathrm{mg}, 0.056$ mmol). The mixture was allowed to stir for $18 \mathrm{hr}$ and then diluted with $\mathrm{NaHCO}_{3}(2 \mathrm{~mL}$ of a sat. aq. solution) and extracted with pentane $(3 \times 3 \mathrm{~mL})$. The combined organic layers were dried over anhydrous $\mathrm{MgSO}_{4}$, filtered and concentrated in vacuo. Chromatography on silica (5\% EtOAc / Pentane) gave $18.1 \mathrm{mg}$ of the silyl ether $\mathbf{2 0}$ as a clear colorless oil (90\%).

Data for 20:

$\mathbf{R}_{f}=0.50$ (5\% EtOAc: $95 \%$ pentane).

IR (film): 3076, 3031, 2952, 2929, 2867, 1641, 1455, 1372, 1307, 1256, 1140, $1101 \mathrm{~cm}^{-1}$.

${ }^{1} \mathbf{H}$ NMR $\left(500 \mathrm{MHz}, \mathrm{CDCl}_{3}\right): \delta 7.30(5 \mathrm{H}, \mathrm{m}), 5.77(1 \mathrm{H}, \mathrm{ddt}, J=10.5,14.4,7.1 \mathrm{~Hz}), 4.98(2 \mathrm{H}$, m), $4.35(1 \mathrm{H}, \mathrm{dd}, J=5.8,8.6 \mathrm{~Hz}), 3.96(1 \mathrm{H}$, ddd, $J=2.7,11.6,12.6 \mathrm{~Hz}), 3.84(1 \mathrm{H}, \mathrm{ddt}, J=4.6$, 7.7, $5.6 \mathrm{~Hz}), 3.74(1 \mathrm{H}$, ddd, $J=1.6,5.4,11.6 \mathrm{~Hz}), 3.57(1 \mathrm{H}$, dddd, $J=2.5,3.7,8.6,11.3 \mathrm{~Hz})$, 
$3.29(1 \mathrm{H}, \mathrm{ddd}, J=6.3,7.4,9.3 \mathrm{~Hz}), 3.24(1 \mathrm{H}, \mathrm{ddd}, J=5.5,6.7,9.2 \mathrm{~Hz}), 2.48(1 \mathrm{H}$, ddd, $J=2.1$, 3.6, $13.5 \mathrm{~Hz}), 2.41(1 \mathrm{H}, \mathrm{dsept}, J=2.0,7.0 \mathrm{~Hz}), 2.15(3 \mathrm{H}, \mathrm{m}), 1.40-1.77(8 \mathrm{H}, \mathrm{m}), 1.29(1 \mathrm{H}, \mathrm{ddt}$, $J=1.7,12.9,2.5 \mathrm{~Hz}), 1.16(1 \mathrm{H}, \mathrm{ddd}, J=2.0,4.8,11.8 \mathrm{~Hz}), 0.93(3 \mathrm{H}, \mathrm{d}, J=7.0 \mathrm{~Hz}), 0.89(3 \mathrm{H}, \mathrm{d}$, $J=7.0 \mathrm{~Hz}), 0.85(9 \mathrm{H}, \mathrm{s}), 0.85(1 \mathrm{H}, \mathrm{m}), 0.83(3 \mathrm{H}, \mathrm{d}, J=6.7 \mathrm{~Hz}), 0.62(1 \mathrm{H}, \mathrm{dd}, J=12.4,13.5$ $\mathrm{Hz}), 0.04(3 \mathrm{H}, \mathrm{s}), 0.02(3 \mathrm{H}, \mathrm{s})$.

${ }^{13}$ C NMR (125 MHz, $\left.\mathrm{CDCl}_{3}\right): \delta 142.0,135.0,128.3$ (x2), 127.7, 127.4 (x2), 116.8, 100.5, 78.7, $68.9,64.9,64.6,58.9,51.2,44.8,42.2,37.4,36.9,34.9,31.6,29.1,25.8$ (x3), 24.4, 23.8, 22.0, $21.9,19.1,18.0,-4.4,-4.7$.

HRMS: $\left[\mathrm{M}^{+}\right]$Calculated for $\mathrm{C}_{33} \mathrm{H}_{56} \mathrm{O}_{4} \mathrm{Si}: 544.3948$. Found: 544.3941 .

$[\alpha]_{D}^{24}=-4.5^{\circ}\left(c 1.80, \mathrm{CHCl}_{3}\right)$.
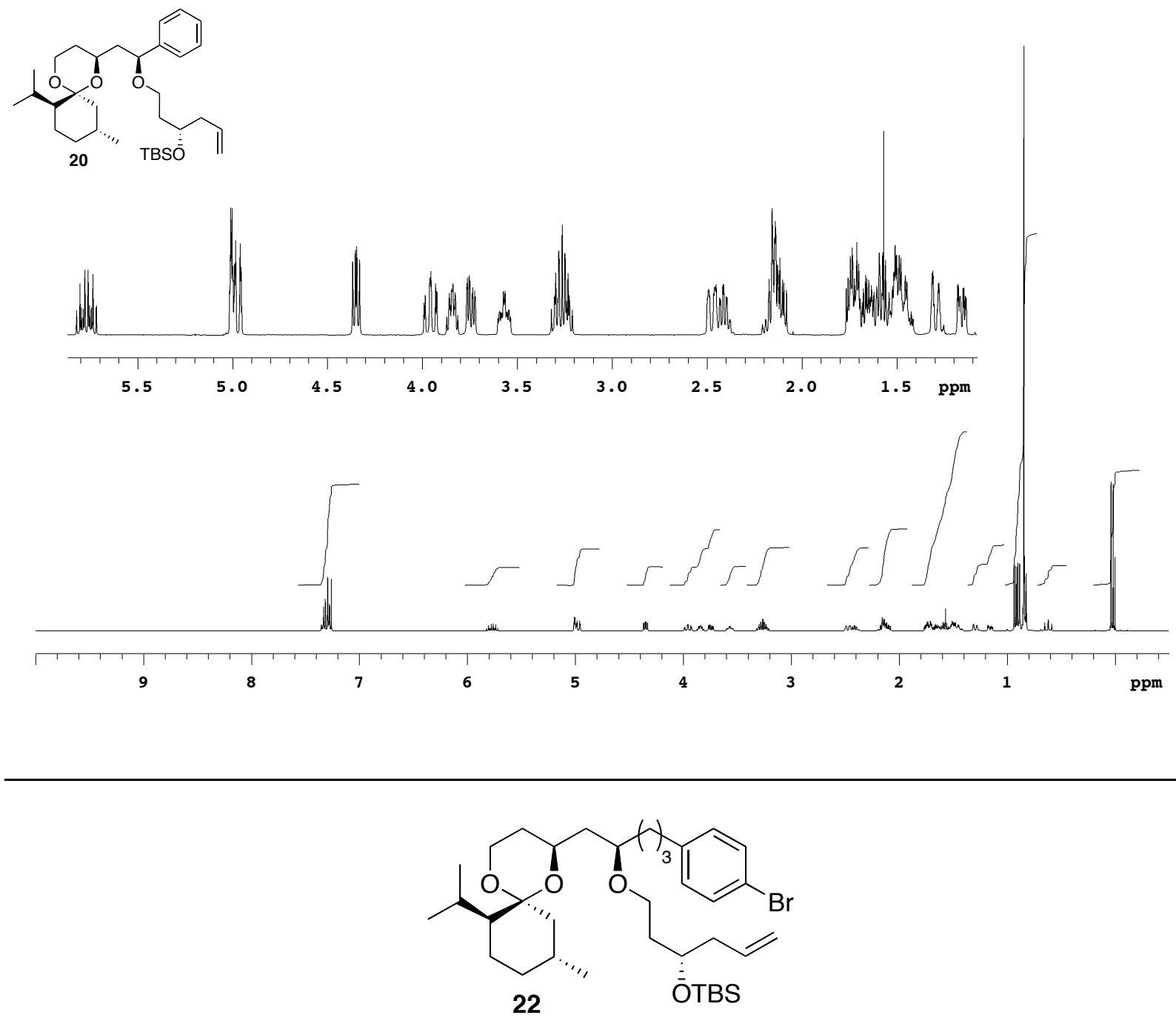

A stock solution of (-)-Ipc ${ }_{2} \mathrm{~B}$ (allyl) was prepared by dissolving (-)-Ipc ${ }_{2} \mathrm{BOMe}(157 \mathrm{mg}$, $0.496 \mathrm{mmol})$ in $\mathrm{Et}_{2} \mathrm{O}(1.19 \mathrm{~mL})$ in $10 \mathrm{~mL}$ round bottom flask. The reaction flask was immersed in a dry ice/acetone bath and allylmagnesium bromide, in the form of a $1 \mathrm{M}$ solution in THF 
(421 $\mu \mathrm{L}, 0.421 \mathrm{mmol}$ ), was added drop-wise. The dry ice/acetone bath was then removed and the solution was allowed to warm to rt. After stirring for $1 \mathrm{~h}$ at $\mathrm{rt}$ stirring was stopped. In a separate $25 \mathrm{~mL}$ round bottom flask $18(55 \mathrm{mg}, 0.108 \mathrm{mmol})$ was dissolved in $\mathrm{Et}_{2} \mathrm{O}$ (3.3 mL). This flask was then cooled in a dry ice/acetone bath and the solution of (-)-Ipc ${ }_{2} \mathrm{~B}$ (allyl) $(632 \mu \mathrm{L}, 0.15$ $\mathrm{mmol}$ ) was added drop-wise. Upon complete addition the reaction was allowed to proceed for 2 $\mathrm{h}$ immersed in the dry ice/acetone bath. $3 \mathrm{M} \mathrm{NaOH}_{\text {(aq.) }}(108 \mu \mathrm{L})$ and $30 \%$ (w/w) $\mathrm{H}_{2} \mathrm{O}_{2 \text { (aq.) }}(262$ $\mu \mathrm{L})$ were then added to the reaction and the cold bath was removed. The reaction was stirred for another $2 \mathrm{~h}$ and excess $\mathrm{H}_{2} \mathrm{O}_{2}$ was quenched by the addition of a saturated solution of $\mathrm{Na}_{2} \mathrm{~S}_{2} \mathrm{O}_{3 \text { (aq.) }}$ $(5 \mathrm{~mL})$. The reaction was diluted with $\mathrm{Et}_{2} \mathrm{O}(30 \mathrm{~mL})$ and then this solution was extracted with brine $(2 \times 40 \mathrm{~mL})$. The organic phase was then dried over $\mathrm{MgSO}_{4}$, which was then removed by vacuum filtration. The filtrate was concentrated and the residue was purified by flash chromatography (silica gel $1 / 4 \mathrm{Et}_{2} \mathrm{O}$ :pentane) to give the homoallylic alcohol as a mixture with the pinanol by-product.

This mixture was then dissolved in DMF $(829 \mu \mathrm{L})$ in a $25 \mathrm{~mL}$ round bottom flask. To this solution was added TBSCl $(47 \mathrm{mg}, 0.315 \mathrm{mmol})$ in one portion followed by imidazole $(25$ $\mathrm{mg}, 0.368 \mathrm{mmol}$ ), also added in one portion. After $1 \mathrm{~h}$ the reaction was not complete so additional TBSCl (31 mg, $0.206 \mathrm{mmol})$ and imidazole $(17 \mathrm{mg}, 0.026 \mathrm{mmol}$ ) were added and the reaction was stirred for an additional $2 \mathrm{~h}$. The reaction was then diluted with pentane $(40 \mathrm{~mL})$ and this solution was extracted with saturated $\mathrm{NH}_{4} \mathrm{Cl}_{(\text {aq. }}(3 \mathrm{x} 40 \mathrm{~mL})$ and brine $(1 \mathrm{x} 40 \mathrm{~mL})$. The organic phase was then dried over $\mathrm{MgSO}_{4}$, which was subsequently removed by vacuum filtration. The filtrate was concentrated in vacuo and $\mathbf{2 2}$ was isolated from the residue by flash chromatography (silica gel 1/9 $\mathrm{Et}_{2} \mathrm{O}$ :pentane) as a clear oil (56 mg, 78\% over 2 steps).

Data for 4.45:

$\mathbf{R}_{f}=0.52\left(10 \% \mathrm{Et}_{2} \mathrm{O} /\right.$ pentane $)$.

${ }^{1}$ H NMR (500 MHz, $\left.\mathrm{CDCl}_{3}\right): \delta 7.39(2 \mathrm{H}, \mathrm{d}, J=8.5 \mathrm{~Hz}), 7.04(2 \mathrm{H}, \mathrm{d}, J=8.5 \mathrm{~Hz}), 5.80(1 \mathrm{H}, \mathrm{m})$, $5.03(2 \mathrm{H}, \mathrm{m}), 4.06(1 \mathrm{H}, \mathrm{td}, J=2.5,12.5 \mathrm{~Hz}), 3.81(3 \mathrm{H}, \mathrm{m}), 3.47(1 \mathrm{H}, \mathrm{ddd}, J=7,9,14), 3.42$ $(1 \mathrm{H}$, sept, $J=4 \mathrm{~Hz}), 3.37(1 \mathrm{H}, \mathrm{m}), 2.66(1 \mathrm{H}, \mathrm{dq}, J=1.5,13.5 \mathrm{~Hz}), 2.56(2 \mathrm{H}, \mathrm{m}), 2.40(1 \mathrm{H}$, dsept, $J=7,2 \mathrm{~Hz}), 2.21(2 \mathrm{H}, \mathrm{m}), 1.33-1.82(14 \mathrm{H}, \mathrm{m}), 1.17(1 \mathrm{H}, \mathrm{dq}, J=2,12.5 \mathrm{~Hz}), 0.85-0.91$ $(19 \mathrm{H}, \mathrm{m}), 0.68(1 \mathrm{H}, \mathrm{t}, J=13 \mathrm{~Hz}), 0.04(6 \mathrm{H}, \mathrm{d}, J=5 \mathrm{~Hz})$.

${ }^{13}$ C NMR (125 MHz, $\left.\mathrm{CDCl}_{3}\right): \delta 141.7,135.2,131.5$ (2C), 130.3 (2C), 119.6, 117.1, 100.7, 75.8, $69.5,65.5,64.9,59.3,51.4,42.4,40.8,37.6,37.3,35.7,35.2,33.6,32.2$, 29.4, 27.4, 26.1 (3C), $24.5,24.0,22.5,22.1,19.2,18.3,-4.1,-4.4$.

IR (thin film): 2950, 2865, 1488, 1461, 1374, 1307, 1256, 1218, 1142, 1111, 976, 913, 836, 775 $\mathrm{cm}^{-1}$.

HRMS: $\left[\mathrm{M}^{+}\right]$Calculated for $\mathrm{C}_{36} \mathrm{H}_{61} \mathrm{BrO}_{4} \mathrm{Si}$ : 664.3522; found: 664.3521.

$[\alpha]_{\mathrm{D}}^{23}=+13.2^{\circ}\left(c 2.82, \mathrm{CH}_{2} \mathrm{Cl}_{2}\right)$. 

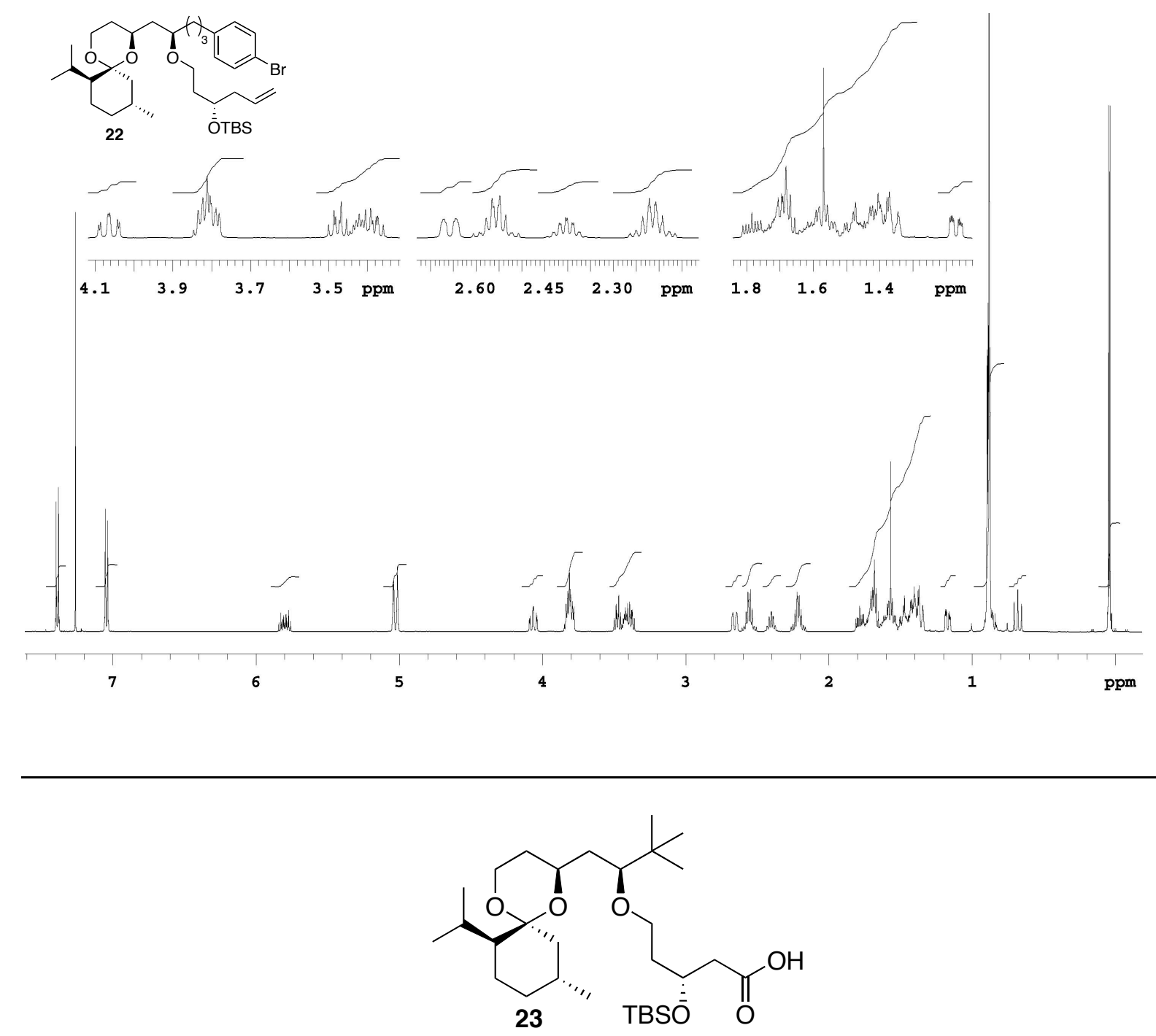

$\mathrm{NaIO}_{4}(121 \mathrm{mg}, 0.566 \mathrm{mmol})$ and $\mathrm{KMnO}_{4}(11 \mathrm{mg}, 0.070 \mathrm{mmol})$ were suspended in $4 \mathrm{~mL}$ of $\mathrm{pH} 7$ phosphate buffer and stirred at $\mathrm{rt}$ for $10 \mathrm{~min}$. This purple suspension was then poured into a solution of the terminal alkene $19(37 \mathrm{mg}, 0.071 \mathrm{mmol})$ in $t$-BuOH $(3.2 \mathrm{~mL})$ using $1.5 \mathrm{~mL}$ phosphate buffer to rinse the remaining suspension into the reaction flask. The mixture was allowed to stir for $1 \mathrm{hr}$ and then solid $\mathrm{Na}_{2} \mathrm{~S}_{2} \mathrm{O}_{3} \cdot 5 \mathrm{H}_{2} \mathrm{O}(60 \mathrm{mg}, 0.242 \mathrm{mmol})$ was added - the purple suspension immediately turned brown. The reaction mixture was diluted with brine (20 $\mathrm{mL})$ and extracted with EtOAc $(3 \times 25 \mathrm{~mL})$. The combined organic layers were dried over anhydrous $\mathrm{Na}_{2} \mathrm{SO}_{4}$, filtered and concentrated. Chromatography on silica (12\% EtOAc / pentane $+1 \% \mathrm{AcOH})$ gave $30.0 \mathrm{mg}$ of the carboxylic acid $\mathbf{2 3}$ as a clear colorless oil (79\%).

Data for 23:

$\mathbf{R}_{f}=0.17$ (15\% EtOAc / hexanes).

IR (thin film): 3313-3303(br), 2953, 2869, 1714, 1455, 1377, 1306, 1257, 1158, 1106, 974, 837, $776 \mathrm{~cm}^{-1}$. 
${ }^{1} \mathbf{H}$ NMR $\left(600 \mathrm{MHz}, \mathrm{CDCl}_{3}\right): \delta 4.28(1 \mathrm{H}, \mathrm{dq}, J=7.1,5.4 \mathrm{~Hz}), 4.07(1 \mathrm{H}, \mathrm{ddd}, J=2.8,11.6,12.6$ $\mathrm{Hz}), 3.82(1 \mathrm{H}, \mathrm{ddd}, J=1.5,5.3,11.6 \mathrm{~Hz}), 3.76(1 \mathrm{H}, \mathrm{dddd}, J=2.5,4.9,7.6,10.7 \mathrm{~Hz}), 3.68(1 \mathrm{H}$, ddd, $J=5.4,6.1,9.6 \mathrm{~Hz}), 3.42(1 \mathrm{H}, \mathrm{ddd}, J=5.0,7.6,9.6 \mathrm{~Hz}), 2.92(1 \mathrm{H}, \mathrm{t}, J=5.0 \mathrm{~Hz}), 2.68(1 \mathrm{H}$, ddd, $J=2.1,3.5,13.5 \mathrm{~Hz}), 2.63(1 \mathrm{H}, \mathrm{dd}, J=5.3,15.1 \mathrm{~Hz}), 2.55(1 \mathrm{H}, \mathrm{dd}, J=5.8,15.1 \mathrm{~Hz}), 2.41$ $(1 \mathrm{H}$, dsept, $J=2.2,7.0 \mathrm{~Hz}), 1.72-1.87(3 \mathrm{H}, \mathrm{m}), 1.64(1 \mathrm{H}, \mathrm{dt}, J=5.0,14.8 \mathrm{~Hz}), 1.39-1.58(6 \mathrm{H}$, $\mathrm{m}), 1.20(1 \mathrm{H}, \mathrm{ddd}, \mathrm{J}=2.2,3.8,12.8 \mathrm{~Hz}), 0.88-0.91(28 \mathrm{H}, \mathrm{m}), 0.70(1 \mathrm{H}, \mathrm{dd}, \mathrm{J}=12.5,13.5 \mathrm{~Hz})$, $0.10(3 \mathrm{H}, \mathrm{s}), 0.095(3 \mathrm{H}, \mathrm{s})$.

${ }^{13} \mathrm{C}$ NMR $\left(100 \mathrm{MHz}, \mathrm{CDCl}_{3}\right) \delta 174.2,100.7,84.5,67.2,67.0,66.6,59.2,51.4,41.7,38.7,37.4$, 37.3, 35.8, 35.0, 31.9, 29.1, 26.1 (x3), 25.7 (x3), 24.3, 23.8, 22.2, 22.0, 19.0, 17.9, -4.7, -5.0.

HRMS: [M+] Calculated for $\mathrm{C}_{30} \mathrm{H}_{58} \mathrm{O}_{6} \mathrm{Si}$ : 542.4003; found: 542.4006 .

$[\alpha]_{D}^{22}=-5.88^{\circ}\left(C 1.67, \mathrm{CH}_{2} \mathrm{Cl}_{2}\right)$.

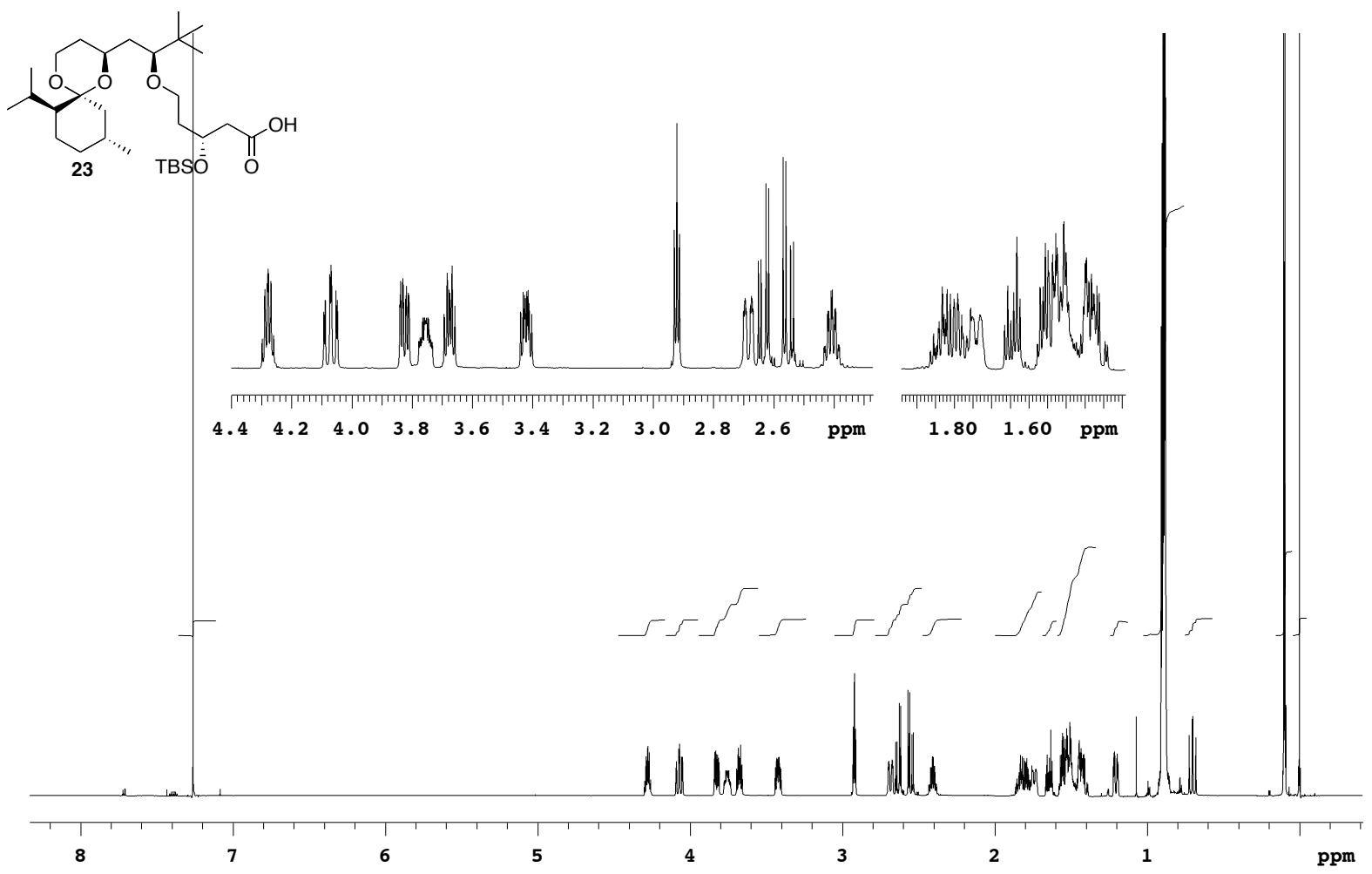

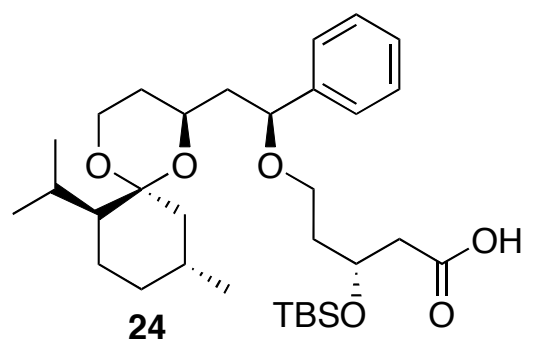


$\mathrm{NaIO}_{4}(57.0 \mathrm{mg}, 0.266 \mathrm{mmol})$ and $\mathrm{KMnO}_{4}(5.0 \mathrm{mg}, 0.032 \mathrm{mmol})$ were suspended in 1.7 $\mathrm{mL}$ of $\mathrm{pH} 7$ phosphate buffer and stirred at $\mathrm{rt}$ for $15 \mathrm{~min}$. This purple suspension was then poured into a solution of the terminal alkene $20(18.0 \mathrm{mg}, 0.0330 \mathrm{mmol})$ in $t$-BuOH $(1.3 \mathrm{~mL})$ using $0.9 \mathrm{~mL}$ phosphate buffer to rinse the remaining suspension into the reaction flask. The mixture was allowed to stir for $15 \mathrm{~min}$ and then solid $\mathrm{Na}_{2} \mathrm{~S}_{2} \mathrm{O}_{3} \cdot 5 \mathrm{H}_{2} \mathrm{O}(22 \mathrm{mg}, 0.089 \mathrm{mmol})$ was added - after $15 \mathrm{sec}$, the purple suspension turned brown. The reaction mixture was diluted with brine $(25 \mathrm{~mL})$ and extracted with EtOAc $(3 \times 33 \mathrm{~mL})$. The combined organic layers were dried over anhydrous $\mathrm{Na}_{2} \mathrm{SO}_{4}$, filtered and concentrated in vacuo. Chromatography on silica (12\% EtOAc / pentane $+1 \% \mathrm{AcOH}$ ) gave $13.0 \mathrm{mg}$ of the carboxylic acid $\mathbf{2 4}$ as a clear colorless oil $(70 \%)$.

Data for 24:

$\mathbf{R}_{f}=0.40(30 \% \mathrm{EtOAc} /$ pentane $+1 \% \mathrm{AcOH})$.

IR (thin film): 3059, 3024, 2952, 2867, 1711, 1454, 1375, 1307, 1257, 1158, 1140, $1100 \mathrm{~cm}^{-1}$.

${ }^{1} \mathbf{H}$ NMR $\left(500 \mathrm{MHz}, \mathrm{CDCl}_{3}\right): \delta 7.31(5 \mathrm{H}, \mathrm{m}), 4.36(1 \mathrm{H}, \mathrm{dd}, J=5.6,9.0 \mathrm{~Hz}), 4.26(1 \mathrm{H}$, quint, $J=$ 5.8), $4.00(1 \mathrm{H}, \mathrm{ddd}, J=2.6,11.7,12.7 \mathrm{~Hz}), 3.74(1 \mathrm{H}, \mathrm{ddd}, J=1.5,5.3,11.6 \mathrm{~Hz}), 3.54(1 \mathrm{H}$, dddd, $J=2.7,3.6,8.9,11.5 \mathrm{~Hz}), 3.32(1 \mathrm{H}$, ddd, $J=5.4,7.2,9.6 \mathrm{~Hz}), 3.24(1 \mathrm{H}$, ddd, $J=5.6,6.4$, $9.5 \mathrm{~Hz}), 2.44$ (4H, m, C11), 2.11 (1H, ddd, $J=5.6,8.8,13.9), 1.74$ (4H, m, C4), 1.62 (1H, ddt, $J$ $=5.4,11.4,12.9), 1.49(3 \mathrm{H}, \mathrm{m}), 1.29(1 \mathrm{H}, \mathrm{ddt}, J=1.6,12.9,2.6 \mathrm{~Hz}), 1.16(1 \mathrm{H}, \mathrm{ddd}, J=2.0,4.2$, $12.5 \mathrm{~Hz}), 0.93(3 \mathrm{H}, \mathrm{d}, J=7.0 \mathrm{~Hz}), 0.89(3 \mathrm{H}, \mathrm{d}, J=7.1 \mathrm{~Hz}), 0.86(1 \mathrm{H}, \mathrm{m}), 0.85(9 \mathrm{H}, \mathrm{s}), 0.82(3 \mathrm{H}$, d, $J=6.7 \mathrm{~Hz}), 0.62(1 \mathrm{H}, \mathrm{dd}, J=12.6,13.4 \mathrm{~Hz}), 0.08(6 \mathrm{H}, \mathrm{s})$.

${ }^{13}$ C NMR (125 MHz, $\left.\mathrm{CDCl}_{3}\right): \delta 175.0,141.4,128.4(\mathrm{x} 2), 127.9,127.4(\mathrm{x} 2), 100.5,79.0,66.7$, 64.5, 64.1, 58.9, 51.2, 44.6, 41.9, 37.4, 37.1, 34.8, 31.6, 29.1, 25.7 (x3), 24.3, 23.8, 21.9 (x2), $19.1,17.9,-4.8,-4.9$.

HRMS: $\left[\mathrm{M}^{+}\right]$Calculated for $\mathrm{C}_{35} \mathrm{H}_{54} \mathrm{O}_{6} \mathrm{Si}$ : 562.3690. Found: 562.3665.

$[\alpha]_{D}^{26}=-5.7^{\circ}\left(c 1.3, \mathrm{CHCl}_{3}\right)$. 

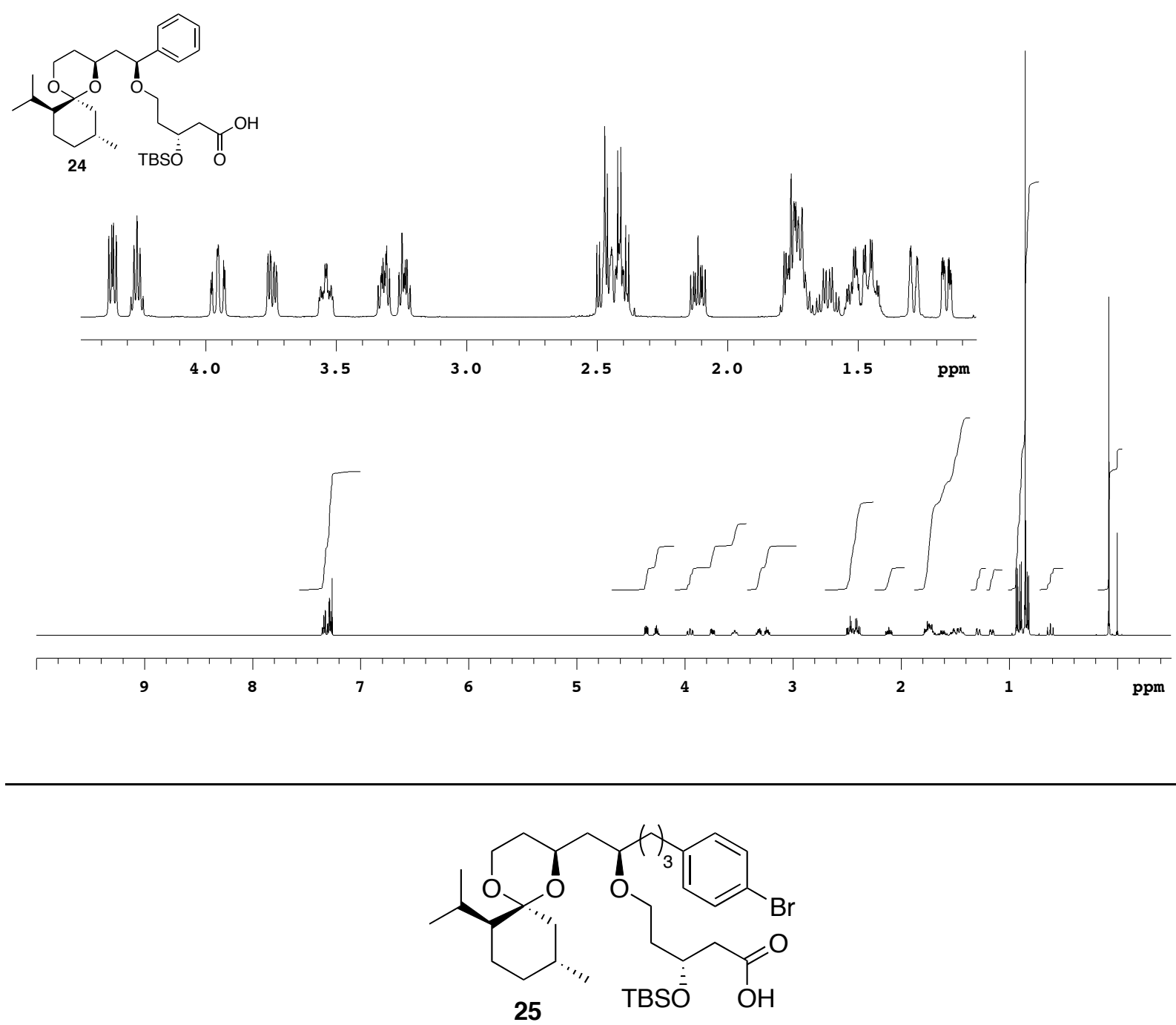

In a $10 \mathrm{~mL}$ round bottom flask $\mathrm{NaIO}_{4}(80 \mathrm{mg}, 0.373 \mathrm{mmol})$ and $\mathrm{KMnO}_{4}(7.4 \mathrm{mg}, 0.047$ mmol) were dissolved in a $\mathrm{pH} 7$ aqueous buffer $(4.3 \mathrm{~mL})$. This purple solution was stirred for 10 min at rt. In a separate $25 \mathrm{~mL}$ round bottom flask $22(31 \mathrm{mg}, 0.047 \mathrm{mmol})$ was dissolved in $t$ $\mathrm{BuOH}(2.2 \mathrm{~mL})$. To this solution was added the first solution of $\mathrm{NaIO}_{4}, \mathrm{KMnO}_{4}$, and $\mathrm{pH} 7$ buffer as a continuous stream (as opposed to drop-wise). The resulting solution was stirred for $3 \mathrm{~h}$ at $\mathrm{rt}$. The reaction was quenched by the addition of $\mathrm{Na}_{2} \mathrm{~S}_{2} \mathrm{O}_{3} \cdot 5 \mathrm{H}_{2} \mathrm{O}$ at $\mathrm{rt}(31 \mathrm{mg}, 0.128 \mathrm{mmol})$, causing the reaction to change color, from purple to brown. The reaction was stirred for another $10 \mathrm{~min}$ at $\mathrm{rt}$ and then the reaction was extracted with EtOAc $(3 \times 15 \mathrm{~mL})$ and the combined organic phases were dried over $\mathrm{MgSO}_{4}$. Removal of the $\mathrm{MgSO}_{4}$ by vacuum filtration and then concentration of the filtrate in vacuo yielded a residue from which $\mathbf{2 5}$ was isolated by flash chromatography (silica gel 1/4 EtOAc:pentane $+1 \%$ acetic acid) as a clear oil $(24 \mathrm{mg}, 75 \%)$.

Data for 25:

$\mathbf{R}_{f}=0.59$ (20\% EtOAc / pentane $+1 \%$ acetic acid). 
${ }^{1}$ H NMR $\left(500 \mathrm{MHz}, \mathrm{CDCl}_{3}\right): \delta 7.39(2 \mathrm{H}, \mathrm{d}, J=8 \mathrm{~Hz}), 7.04(2 \mathrm{H}, \mathrm{d}, J=8.5 \mathrm{~Hz}), 4.25(1 \mathrm{H}$, pent, $J=5.5 \mathrm{~Hz}), 4.06(1 \mathrm{H}, \mathrm{td}, J=2.5,12.5 \mathrm{~Hz}), 3.80(1 \mathrm{H}, \mathrm{ddd}, J=1.5,5.5,11.5 \mathrm{~Hz}), 3.77(1 \mathrm{H}, \mathrm{m})$, $3.50(1 \mathrm{H}, \mathrm{dt}, J=6,9.5 \mathrm{~Hz}), 3.37-3.46(2 \mathrm{H}, \mathrm{m}), 2.65(1 \mathrm{H}, \mathrm{ddd}, J=2,3,13.5 \mathrm{~Hz}), 2.49-2.61(4 \mathrm{H}$, $\mathrm{m}), 2.40(1 \mathrm{H}$, dsept, $J=7,1.5 \mathrm{~Hz}), 1.66-1.87(4 \mathrm{H}, \mathrm{m}), 1.31-1.65(10 \mathrm{H}, \mathrm{m}), 1.17(1 \mathrm{H}, \mathrm{dq}, J=2,7$ $\mathrm{Hz}), 0.85-0.90(19 \mathrm{H}, \mathrm{m}), 0.68(1 \mathrm{H}, \mathrm{t}, J=13 \mathrm{~Hz}), 0.07(6 \mathrm{H}, \mathrm{d}, J=3 \mathrm{~Hz})$.

${ }^{13}$ C NMR (125 MHz, $\left.\mathrm{CDCl}_{3}\right): \delta$ 175.4, 141.3, 131.3 (2 C), 130.1 (2 C), 119.4, 100.4, 76.1, 67.0, 64.6, 64.56, 59.1, 51.2, 42.1, 40.4, 37.3 (2 C), 35.4, 34.9, 33.3, 32.0, 29.2, 27.0, 25.7 (3 C), 24.3, 23.7, 22.3, 21.9, 18.9, 17.9, -4.7, -4.9.

IR (thin film): 2951, 2929, 2866, 1711, 1456, 1374, 1306, 1257, 1218, 1142, 1107, 976, 836, $776 \mathrm{~cm}^{-1}$.

HRMS: [ $\left.\mathrm{M}^{+}\right]$Calculated for $\mathrm{C}_{35} \mathrm{H}_{59} \mathrm{BrO}_{6} \mathrm{Si}$ : 682.3264; found: 682.3273 .

$[\alpha]_{\mathrm{D}}^{24}=+5.1^{\circ}\left(c 2.48, \mathrm{CH}_{2} \mathrm{Cl}_{2}\right)$.

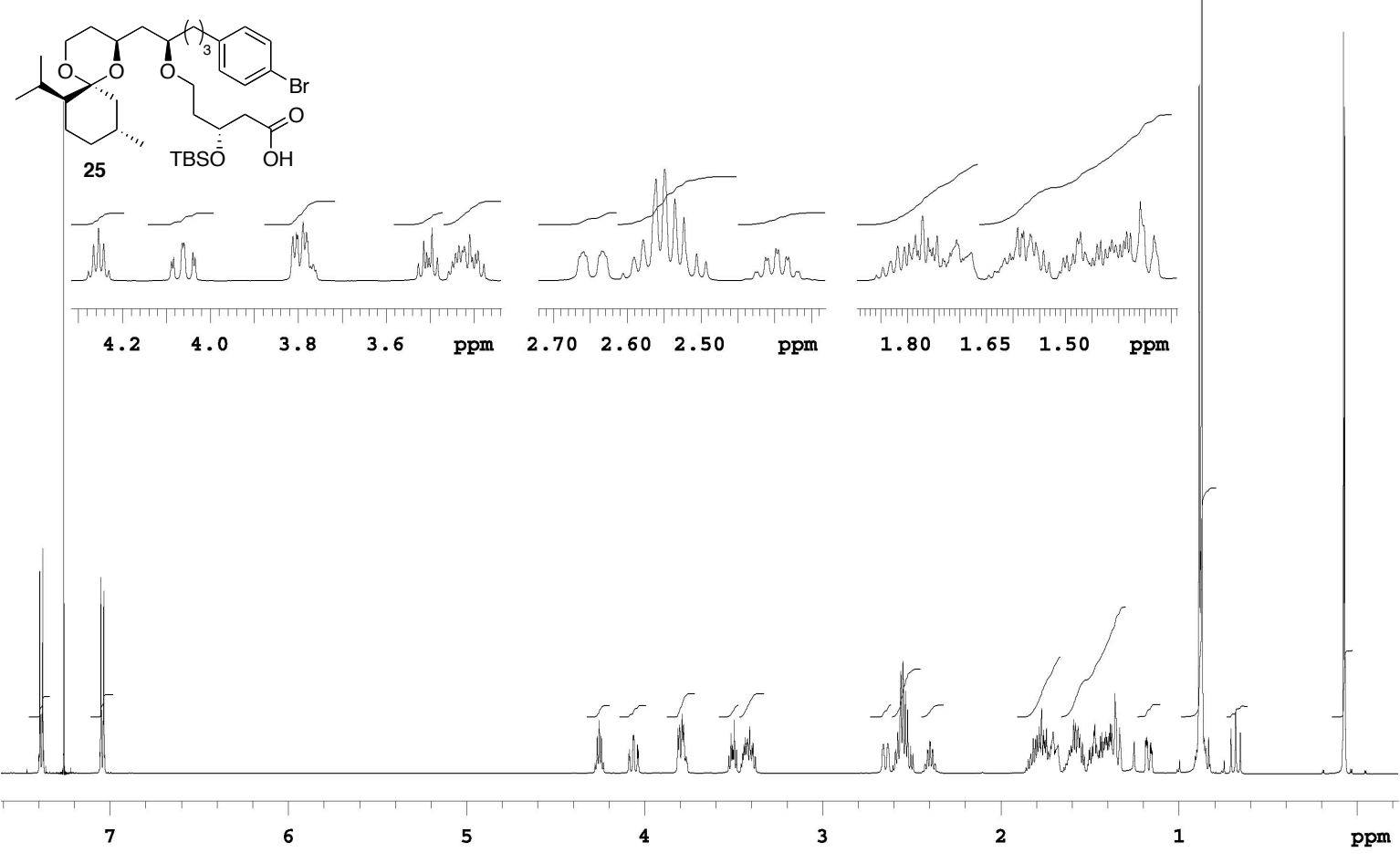




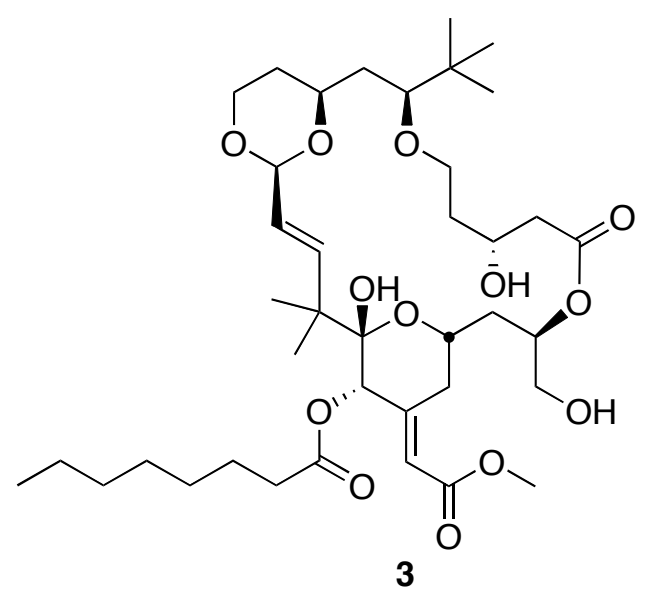

To a solution of carboxylic acid $23(17.7 \mathrm{mg}, 0.0326 \mathrm{mmol})$ in $\mathrm{CH}_{2} \mathrm{Cl}_{2}(0.4 \mathrm{~mL})$, under $\mathrm{N}_{2}$ at rt, was added $i-\operatorname{Pr}_{2} \operatorname{EtN}(10.0 \mu \mathrm{L}, 0.0575 \mathrm{mmol})$ and the solution was stirred for $2 \mathrm{~min}$. PyBroP (20.8 $\mathrm{mg}, 0.0446 \mathrm{mmol}$ ) was then added and the solution stirred for $5 \mathrm{~min}$. A solution of recognition domain $26(20.0 \mathrm{mg}, 0.0334 \mathrm{mmol})$ in $\mathrm{CH}_{2} \mathrm{Cl}_{2}(0.4 \mathrm{~mL})$ was then added to the reaction mixture immediately followed by DMAP $(6.0 \mathrm{mg}, 0.050 \mathrm{mmol})$. The mixture was allowed to stir for $3 \mathrm{hr}$ and then the solvent was removed under a stream of $\mathrm{N}_{2}$. The residue was chromatographed on silica (10\% EtOAc / pentane) to give $33.0 \mathrm{mg}$ of colorless oil. This material was observed (by TLC) to slowly decompose when dissolved in $\mathrm{CDCl}_{3}$ (neutralized with $\mathrm{Na}_{2} \mathrm{CO}_{3}$ ); it was therefore used immediately in the next reaction.

To a solution of the coupled ester in THF $(8 \mathrm{~mL})$, under $\mathrm{N}_{2}$ at $0^{\circ} \mathrm{C}$ in a polypropylene vial, was added $\mathrm{HF} \bullet$ pyridine $(1.7 \mathrm{~mL})$ drop-wise via syringe over $5 \mathrm{~min}$. The solution was allowed to warn to rt and stir for $24 \mathrm{hr}$. The reaction solution was cooled to $0^{\circ} \mathrm{C}$ and then quenched slowly by addition of water $(10 \mathrm{~mL})$ followed by slow addition of $\mathrm{NaHCO}_{3}(20 \mathrm{~mL}$ of a sat. aq. solution, vigorous bubbling!). The aqueous phase was extracted with EtOAc $(3 \times 40 \mathrm{~mL})$. The combined organic layers were dried over anhydrous $\mathrm{Na}_{2} \mathrm{SO}_{4}$, filtered and concentrated in vacuo. Chromatography on silica (50\% EtOAc / Pentane) gave $8.5 \mathrm{mg}$ of the completed analog 3 as a white amorphous solid (35\% over 2 steps).

Data for 3:

$\mathbf{R}_{f}=0.58$ (80\% EtOAc, $20 \%$ pentane).

IR (thin film): 3462 (br), 3368 (br), 2953, 2924, 2854, 1727, 1665, 1459, 1375, 1288, 1260, $1228,1177,1155,1133,1099,1078,1021,1007 \mathrm{~cm}^{-1}$.

${ }^{1} \mathbf{H}$ NMR $\left(500 \mathrm{MHz}, \mathrm{C}_{6} \mathrm{D}_{6}\right): \delta 6.46(1 \mathrm{H}, \mathrm{d} J=15.9 \mathrm{~Hz}), 6.41(1 \mathrm{H}, \mathrm{d} J=2.0 \mathrm{~Hz}), 5.85(1 \mathrm{H}, \mathrm{dd}, \mathrm{J}$ = 7.2, $15.9 \mathrm{~Hz}), 5.59(1 \mathrm{H}, \mathrm{m}), 5.58(1 \mathrm{H}, \mathrm{s}), 5.48(1 \mathrm{H}, \mathrm{d}, \mathrm{J}=7.2 \mathrm{~Hz}), 4.94(1 \mathrm{H}$, br. s) $4.36(1 \mathrm{H}$, $\mathrm{tt}, \mathrm{J}=2.7,11.3 \mathrm{~Hz}), 4.28(1 \mathrm{H}, \mathrm{dd}, \mathrm{J}=2.5,13.6 \mathrm{~Hz}), 4.19(2 \mathrm{H}, \mathrm{m}), 3.94(1 \mathrm{H}, \mathrm{dd} \mathrm{J}=4.2,10.8 \mathrm{~Hz})$, $3.76(1 \mathrm{H}, \mathrm{dt}, \mathrm{J}=2.4,12.1 \mathrm{~Hz}), 3.60(1 \mathrm{H}, \mathrm{m}), 3.53(1 \mathrm{H}, \mathrm{dd}, \mathrm{J}=3.6,11.9 \mathrm{~Hz}), 3.35(2 \mathrm{H}, \mathrm{m}), 3.27$ $(1 \mathrm{H}, \mathrm{m}), 3.23(3 \mathrm{H}, \mathrm{s}), 2.55(1 \mathrm{H}, \mathrm{dd}, \mathrm{J}=4.8,10.3 \mathrm{~Hz}), 2.42(1 \mathrm{H}, \mathrm{t}, \mathrm{J}=12.7 \mathrm{~Hz}), 2.31(1 \mathrm{H}, \mathrm{ddd}, \mathrm{J}$ $=2.1,11.3,13.9 \mathrm{~Hz}), 2.05(3 \mathrm{H}, \mathrm{m}), 1.74(3 \mathrm{H}, \mathrm{m}), 1.50(4 \mathrm{H}, \mathrm{m}), 1.45(3 \mathrm{H}, \mathrm{s}), 1.27(1 \mathrm{H}, \mathrm{qd}, \mathrm{J}=$ 2.6, $14.9 \mathrm{~Hz}), 1.19(2 \mathrm{H}, \mathrm{m}), 1.12(3 \mathrm{H}, \mathrm{s}), 1.09(6 \mathrm{H}, \mathrm{m}), 0.88(1 \mathrm{H}, \mathrm{m}), 0.84(3 \mathrm{H}, \mathrm{t}, \mathrm{J}=7.4 \mathrm{~Hz})$, $0.81(1 \mathrm{H}, \mathrm{m}), 0.75(9 \mathrm{H}, \mathrm{s})$. 
${ }^{13} \mathrm{C}$ NMR $\left(125 \mathrm{MHz}, \mathrm{CDCl}_{3}\right): \delta 172.4,172.1,166.8,151.4,141.8,127.0,119.9,101.7,98.7$, 86.6, 74.0, 73.6, 71.1, 69.7, 68.1, 66.4, 65.6, 64.6, 51.1, 44.9, 42.8, 38.2, 36.4, 35.8, 34.8, 34.5, $32.5,31.6,31.0,29.0,28.8,26.6(x 3), 24.6,23.8,22.5,19.4,14.1$.

HRMS: $\left[\mathrm{M}^{+} \mathrm{Na}\right]$ Calculated for $\mathrm{C}_{39} \mathrm{H}_{64} \mathrm{O}_{13} \mathrm{Na}$ : 763.4244. Found: 763.4257 .

$[\alpha]_{D}^{29}=-13.3^{\circ}\left(c 0.78, \mathrm{CHCl}_{3}\right)$.
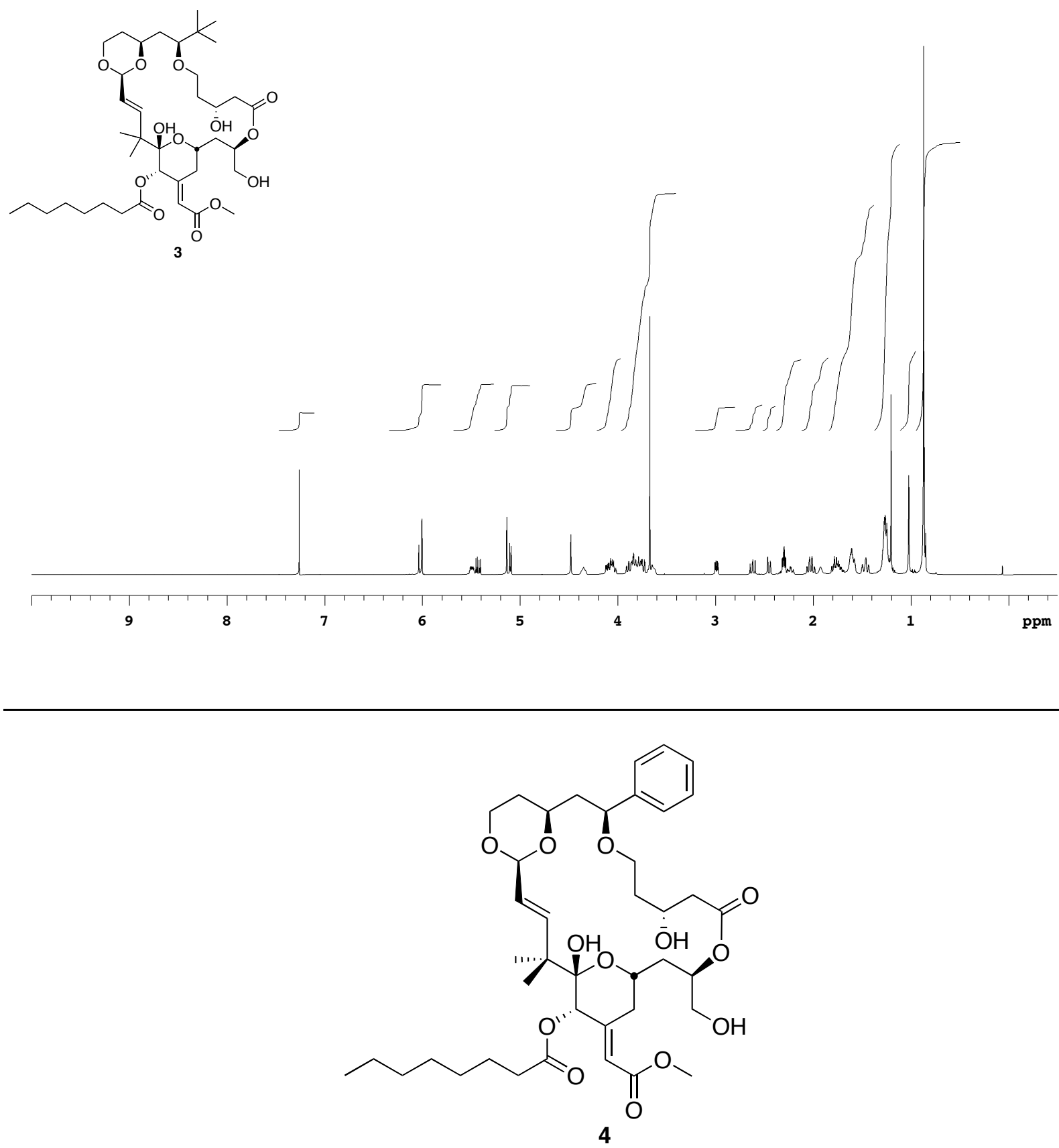

To a solution of carboxylic acid $24(21.0 \mathrm{mg}, 0.0373 \mathrm{mmol})$ in $\mathrm{CH}_{2} \mathrm{Cl}_{2}(0.4 \mathrm{~mL})$, under $\mathrm{N}_{2}$ at rt, was added $i-\mathrm{Pr}_{2} \mathrm{EtN}(12.0 \mu \mathrm{L}, 0.0668 \mathrm{mmol})$ and the solution was stirred for $3 \mathrm{~min}$. PyBroP 
(23.0 $\mathrm{mg}, 0.0501 \mathrm{mmol})$ was then added and the solution stirred for $6 \mathrm{~min}$. A solution of recognition domain $26(20.0 \mathrm{mg}, 0.0334 \mathrm{mmol})$ in $\mathrm{CH}_{2} \mathrm{Cl}_{2}(0.4 \mathrm{~mL})$ was then added to the reaction mixture immediately followed by DMAP $(6.0 \mathrm{mg}, 0.050 \mathrm{mmol})$. The mixture was allowed to stir for $4 \mathrm{hr}$ and then the solvent was removed under a stream of $\mathrm{N}_{2}$. The residue was directly chromatographed on silica (15\% EtOAc / pentane) to give $29.4 \mathrm{mg}$ of the coupled ester as a colorless oil.

To a solution of the coupled ester in THF $(7 \mathrm{~mL})$, under $\mathrm{N}_{2}$ at $0^{\circ} \mathrm{C}$ in a polypropylene vial, was added HF•pyridine $(1.5 \mathrm{~mL})$. The solution was allowed to warn to $\mathrm{rt}$ and stir for $7.5 \mathrm{hr}$. The reaction solution was transferred to a separatory funnel using EtOAc. Water $(15 \mathrm{~mL})$ was added followed by slow addition of $\mathrm{NaHCO}_{3}(50 \mathrm{~mL}$ of a sat. aq. solution, vigorous bubbling!). The aqueous phase was extracted with EtOAc $(3 \times 33 \mathrm{~mL})$. The combined organic layers were dried over anhydrous $\mathrm{Na}_{2} \mathrm{SO}_{4}$, filtered and concentrated in vacuo. Chromatography on silica $(60 \%$ EtOAc / pentane) gave $15.7 \mathrm{mg}$ of the completed analog 4 as a white amorphous solid (62\% over 2 steps).

Data for 4:

$\mathbf{R}_{f}=0.55$ (80\% EtOAc, $20 \%$ pentane).

IR (film): 3466, 3325 (br), 2929, 2859, 1728, 1667, 1435, 1404, 1377, 1362, 1286, 1259, 1229, $1158,1135,1104,1062,1024,1005 \mathrm{~cm}^{-1}$.

${ }^{1} \mathbf{H}$ NMR (500 MHz, $\left.\mathrm{CDCl}_{3}\right): \delta 7.30(5 \mathrm{H}, \mathrm{m}), 6.02(1 \mathrm{H}, \mathrm{d}, J=16.0 \mathrm{~Hz}), 6.01(1 \mathrm{H}, \mathrm{d}, J=2.1 \mathrm{~Hz})$, $5.46(1 \mathrm{H}, \mathrm{dd}, J=7.4,16.0 \mathrm{~Hz}), 5.41(1 \mathrm{H}, \mathrm{m}), 5.16(1 \mathrm{H}, \mathrm{s}), 5.13(1 \mathrm{H}, \mathrm{s}), 5.11(1 \mathrm{H}, \mathrm{d}, J=7.4 \mathrm{~Hz})$, $4.43(1 \mathrm{H}, \mathrm{d}, J=11.5 \mathrm{~Hz}), 4.36(1 \mathrm{H}, \mathrm{dd}, J=2.5,11.3 \mathrm{~Hz}), 4.29(1 \mathrm{H}, \mathrm{dt}, J=2.5,11.3 \mathrm{~Hz}), 4.08$ $(3 \mathrm{H}, \mathrm{m}), 3.89(2 \mathrm{H}, \mathrm{m}), 3.73(1 \mathrm{H}, \mathrm{m}), 3.68(3 \mathrm{H}, \mathrm{s}), 3.66(1 \mathrm{H}, \mathrm{m}), 3.44(1 \mathrm{H}, \mathrm{m}), 3.31(1 \mathrm{H}, \mathrm{ddd}, J=$ 2.7, 9.6, $13.4 \mathrm{~Hz}), 2.58(1 \mathrm{H}, \mathrm{dd}, J=11.4,12.5 \mathrm{~Hz}), 2.52(1 \mathrm{H}, \mathrm{dd}, J=2.8,12.5 \mathrm{~Hz}), 2.31(2 \mathrm{H}$, m), $2.23(1 \mathrm{H}, \mathrm{m}), 2.15(1 \mathrm{H}, \mathrm{m}), 2.04(2 \mathrm{H}, \mathrm{m}), 1.82(1 \mathrm{H}, \mathrm{ddd}, J=2.9,11.3,14.2 \mathrm{~Hz}), 1.76(1 \mathrm{H}$, $\mathrm{dq}, J=5.0,12.8 \mathrm{~Hz}), 1.61(3 \mathrm{H}, \mathrm{m}), 1.48(1 \mathrm{H}, \mathrm{d}(\mathrm{br}), J=14.6 \mathrm{~Hz}), 1.40(1 \mathrm{H}, \mathrm{d}(\mathrm{br}), J=13.7 \mathrm{~Hz})$, $1.26(8 \mathrm{H}, \mathrm{m}), 1.20(3 \mathrm{H}, \mathrm{s}), 1.06(3 \mathrm{H}, \mathrm{s}), 0.87(3 \mathrm{H}, \mathrm{t}, J=7.2 \mathrm{~Hz})$.

${ }^{13}$ C NMR (125 MHz, $\left.\mathrm{CDCl}_{3}\right): \delta 172.4,172.1,167.0,151.6,142.5,141.1,128.7(\mathrm{x} 2), 128.1$, 126.3 (x2), 125.9, 119.9, 102.3, 98.9, 84.1, 75.9, 74.1, 71.6, 68.8, 66.2, 65.72, 65.66, 64.5, 51.1, $45.5,45.1,42.1,35.8,34.6,33.6,32.3,31.6,31.0,29.0,28.9,24.6,24.3,22.5,19.4,14.1$.

HRMS: $\left[\mathrm{M}^{+} \mathrm{Na}\right]$ Calculated for $\mathrm{C}_{41} \mathrm{H}_{60} \mathrm{O}_{13} \mathrm{Na}$ : 783.3932. Found: 783.3925.

$[\alpha]_{D}^{27}=-122.3^{\circ}\left(c 1.17, \mathrm{C}_{6} \mathrm{D}_{6}\right)$ 


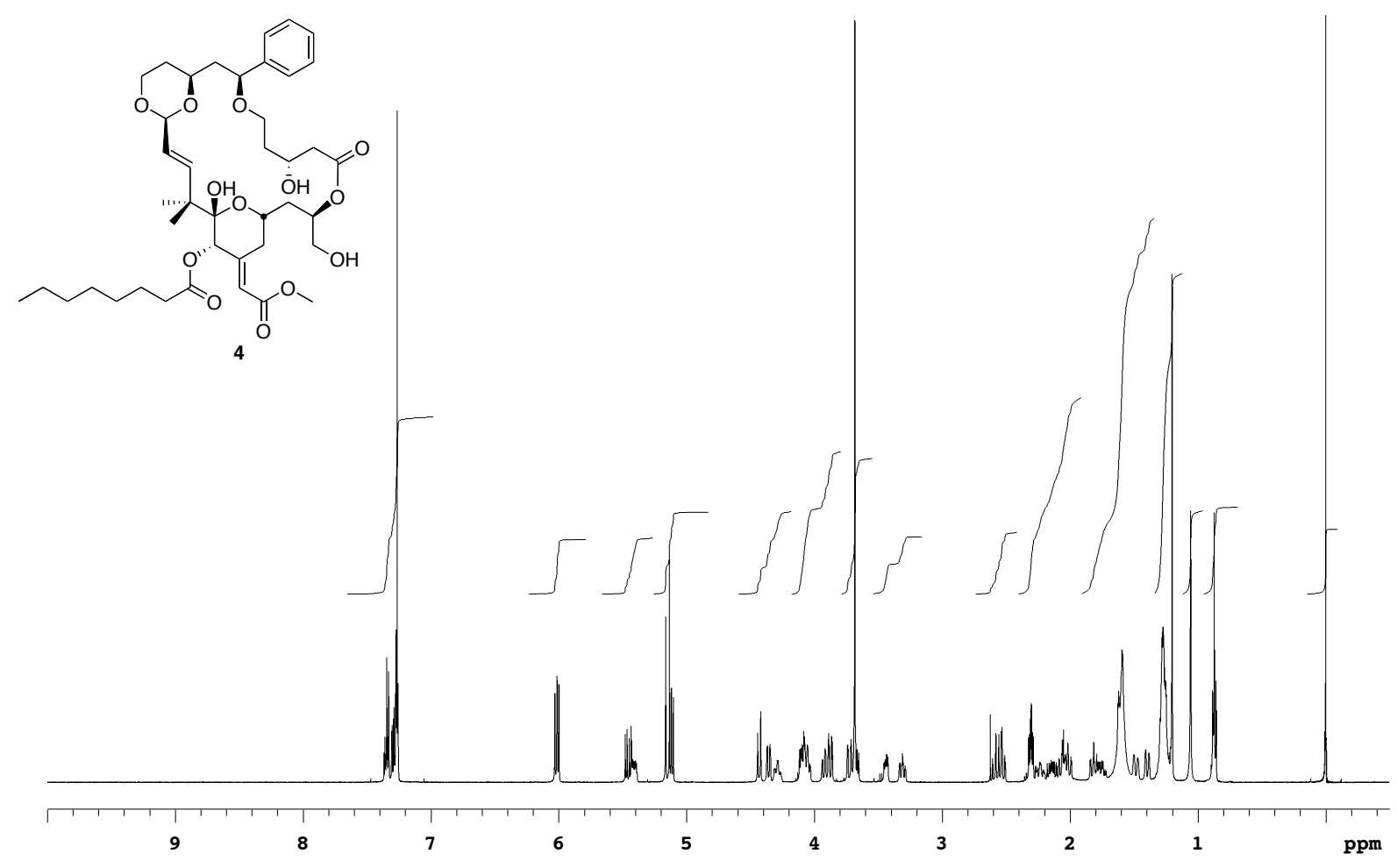

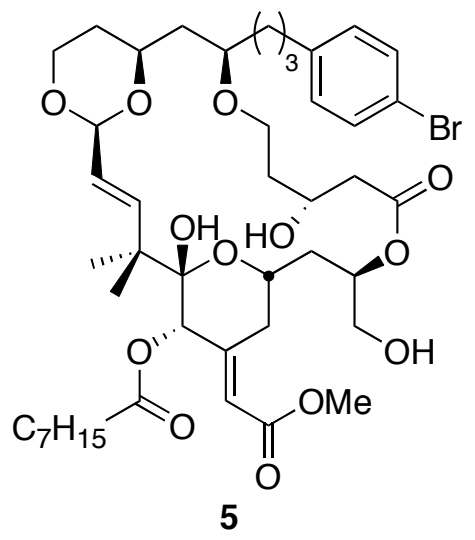

In a $1 \mathrm{~mL}$ round bottom flask $25(19 \mathrm{mg}, 0.028 \mathrm{mmol})$ was dissolved in $\mathrm{CH}_{2} \mathrm{Cl}_{2}(0.69$ $\mathrm{mL})$. To this solution was added $(i \text {-Pr })_{2} \mathrm{EtN}(8.4 \mu \mathrm{L}, 0.048 \mathrm{mmol})$ and PyBroP $(16 \mathrm{mg}, 0.035$ $\mathrm{mmol}$ ), in that order. In a separate $1 \mathrm{~mL}$ round bottom flask $26(15 \mathrm{mg}, 0.025 \mathrm{mmol})$ was dissolved in $\mathrm{CH}_{2} \mathrm{Cl}_{2}(0.69 \mathrm{~mL})$. DMAP (4 mg, $\left.0.035 \mathrm{mmol}\right)$ was added to this solution of 26 in $\mathrm{CH}_{2} \mathrm{Cl}_{2}$ and the entire solution was added to the first solution. The reaction was run for $3 \mathrm{~h}$ at $\mathrm{rt}$ and then was concentrated to an oil under a stream of $\mathrm{N}_{2}$. The coupled product was quickly chromatographed (silica gel 3/17 EtOAc:pentane) and the isolated material was then taken on directly to the next reaction.

This material was dissolved in THF $(4.7 \mathrm{~mL})$ in a dry polypropylene tube under a $\mathrm{N}_{2}$ atmosphere. The tube was then placed in an ice/water bath and a $70 \% \mathrm{w} / \mathrm{w}$ solution of HF•pyridine $(0.919 \mathrm{~mL}, 44.88 \mathrm{mmol}$ of $\mathrm{HF})$ was added drop-wise over $5 \mathrm{~min}$. The ice/water bath 
was removed and the reaction was stirred at $\mathrm{rt}$ for $5 \mathrm{~h}$. The reaction was diluted with EtOAc (10 $\mathrm{mL})$ and then quenched by the slow, drop-wise addition of saturated $\mathrm{NaHCO}_{3 \text { (aq.) }}(10 \mathrm{~mL})$ over $10 \mathrm{~min}$. The aqueous phase was then extracted with EtOAc $(2 \times 30 \mathrm{~mL})$ and the combined organic phases were dried over $\mathrm{MgSO}_{4}$. The $\mathrm{MgSO}_{4}$ was subsequently removed by vacuum filtration. The filtrate was evaporated to a crude residue, from which $\mathbf{5}$ was isolated by flash chromatography (silica gel 4/1 EtOAc:pentane) as a white amorphous solid (14 mg, 84\%).

Data for 5:

$\mathbf{R}_{f}=0.47$ (80\% EtOAc / pentane).

${ }^{1} \mathbf{H}$ NMR $\left(500 \mathrm{MHz}, \mathrm{CDCl}_{3}\right): \delta 7.39(2 \mathrm{H}, \mathrm{d}, J=6.5 \mathrm{~Hz}), 7.03(2 \mathrm{H}, \mathrm{d}, J=8.5 \mathrm{~Hz}), 5.99(1 \mathrm{H}, \mathrm{d}, J$ $=2 \mathrm{~Hz}), 5.96(1 \mathrm{H}, \mathrm{d}, J=16 \mathrm{~Hz}), 5.39(1 \mathrm{H}, \mathrm{dd}, J=7.5,16 \mathrm{~Hz}), 5.37(1 \mathrm{H}, \mathrm{m}, J=3 \mathrm{~Hz}), 5.14(1 \mathrm{H}$, s), $5.03(1 \mathrm{H}, \mathrm{d}, J=6.5 \mathrm{~Hz}), 5.02(1 \mathrm{H}, \mathrm{s}), 4.30\left(1 \mathrm{H}, 1 \mathrm{H}_{\mathrm{F}}, \mathrm{m}\right), 4.12(2 \mathrm{H}, \mathrm{m}), 3.93(1 \mathrm{H}, \mathrm{td}, J=3,12$ $\mathrm{Hz}), 3.91(2 \mathrm{H}, \mathrm{m}), 3.78(1 \mathrm{H}, \mathrm{dd}, J=2,14 \mathrm{~Hz}), 3.75(3 \mathrm{H}, \mathrm{s}), 3.71(2 \mathrm{H}, \mathrm{m}, J=6 \mathrm{~Hz}), 3.53(1 \mathrm{H}, \mathrm{m}$, $J=3 \mathrm{~Hz}), 3.31(1 \mathrm{H}, \mathrm{ddd}, J=2.5,9.5,12.5 \mathrm{~Hz}), 2.61(2 \mathrm{H}, \mathrm{m}), 2.53(2 \mathrm{H}, \mathrm{m}), 2.35(2 \mathrm{H}, \mathrm{td}, J=3.5$, $7.5 \mathrm{~Hz}), 2.27(1 \mathrm{H}, \mathrm{m}), 2.02(1 \mathrm{H}, \mathrm{dd}, J=2.5,20.5 \mathrm{~Hz}), 2.02(1 \mathrm{H}, \mathrm{dt}, J=2.5,20 \mathrm{~Hz}), 1.88(1 \mathrm{H}$, ddd, $J=7.5,11.5,15.5 \mathrm{~Hz}), 1.79(1 \mathrm{H}, \mathrm{m}), 1.72(1 \mathrm{H}, \mathrm{dd}, J=5,12.5 \mathrm{~Hz}), 1.44-1.66(7 \mathrm{Hm}), 1.42$ $(1 \mathrm{H}, \mathrm{dd}, J=2,15 \mathrm{~Hz}), 1.35(1 \mathrm{H}, \mathrm{brd}, J=13 \mathrm{~Hz}), 1.20-1.31(8 \mathrm{H}, \mathrm{m}), 1.17(3 \mathrm{H}, \mathrm{s}), 1.02(3 \mathrm{H}, \mathrm{s})$, $0.86(3 \mathrm{H}, \mathrm{t}, J=7 \mathrm{~Hz})$.

${ }^{13}$ C NMR (125 MHz, $\left.\mathrm{CDCl}_{3}\right): \delta 172.4,172.1,167.0,151.6,142.5,140.9,131.4(2 \mathrm{C}), 130.1$ (2C), 125.9, 119.9, 119.6, 102.3, 98.9, 79.9, 76.0, 74.0, 71.6, 68.6, 66.3, 65.7, 64.7, 64.5, 51.1, 45.0, 42.2, 41.2, 35.8, 35.3, 34.6, 33.8, 32.5, 31.6, 31.0, 30.8, 29.0, 28.9, 24.8, 24.6, 24.2, 22.5, $19.3,14.1$.

IR (thin film): 3460, 3338, 2929, 2858, 1737, 1732, 1722, 1716, 1668, 1488, 1463, 1435, 1404, $1381,1362,1285,1258,1230,1160,1139,1102,1084,1010,980,915,885,860,834,807,735$ $\mathrm{cm}^{-1}$.

HRMS: $\left[\mathrm{M}^{+} \mathrm{Na}\right]$ Calculated for $\mathrm{C}_{44} \mathrm{H}_{65} \mathrm{BrO}_{13}+\mathrm{Na}$ : 903.3506 ; found: 903.3517 .

$[\alpha]_{\mathrm{D}}^{26}=-32.8^{\circ}\left(c 1.18, \mathrm{CH}_{2} \mathrm{Cl}_{2}\right)$. 


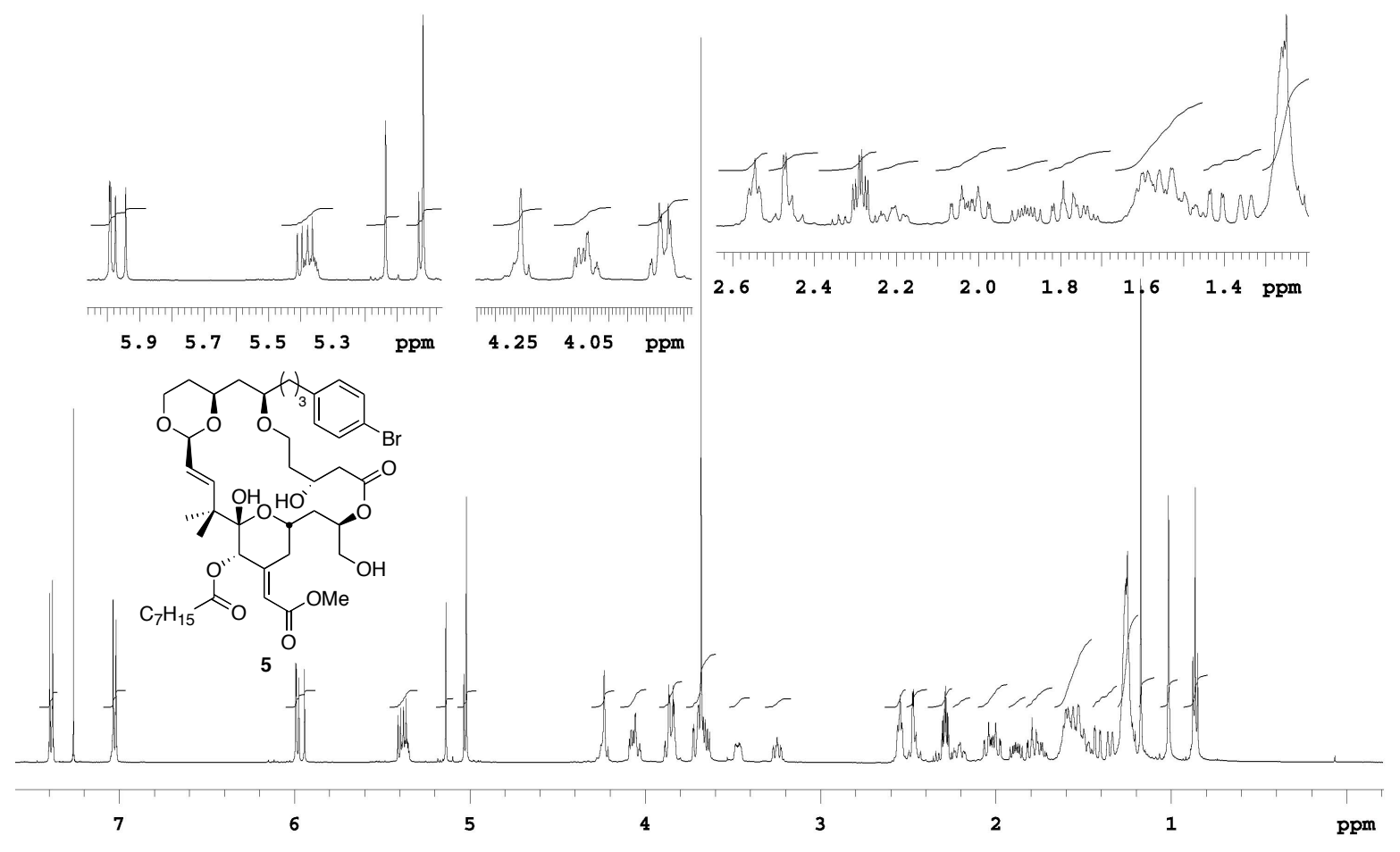

